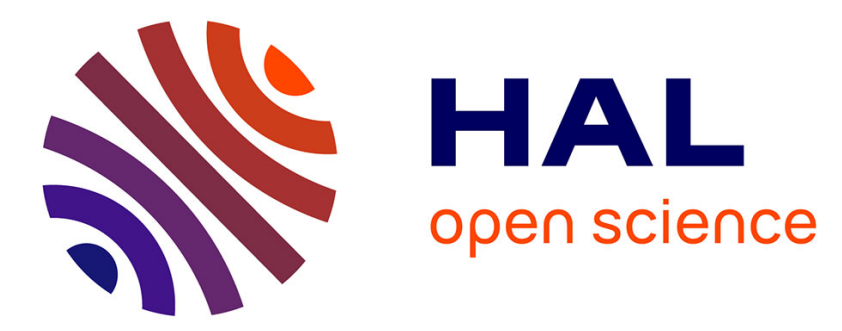

\title{
Asymptotic analysis of shell-like inclusions with high rigidity
}

\author{
Anne-Laure Bessoud, Françoise Krasucki, Michèle Serpilli
}

\section{To cite this version:}

Anne-Laure Bessoud, Françoise Krasucki, Michèle Serpilli. Asymptotic analysis of shell-like inclusions with high rigidity. Journal of Elasticity, 2011, 103 (2), pp.153-172. 10.1007/s10659-010-9278-1 . hal-00790346

\section{HAL Id: hal-00790346 https://hal.science/hal-00790346}

Submitted on 27 Feb 2015

HAL is a multi-disciplinary open access archive for the deposit and dissemination of scientific research documents, whether they are published or not. The documents may come from teaching and research institutions in France or abroad, or from public or private research centers.
L'archive ouverte pluridisciplinaire HAL, est destinée au dépôt et à la diffusion de documents scientifiques de niveau recherche, publiés ou non, émanant des établissements d'enseignement et de recherche français ou étrangers, des laboratoires publics ou privés. 


\title{
Asymptotic analysis of shell-like inclusions with high rigidity
}

Anne-Laure Bessoud

- Françoise Krasucki • Michele Serpilli

Received: date / Accepted: date

\begin{abstract}
We study the problem of an elastic shell-like inclusion with high rigidity in a three-dimensional domain by means of the asymptotic expansion method. The analysis is carried out in a general framework of curvilinear coordinates. After defining a small real adimensional parameter $\varepsilon$, we characterize the limit problems when the rigidity of the inclusion has order of magnitude $\frac{1}{\varepsilon}$ and $\frac{1}{\varepsilon^{3}}$ with respect to the rigidities of the surrounding bodies. Moreover, we prove the strong convergence of the solution of the initial three-dimensional problem towards the solution of the simplified limit problem.
\end{abstract}

Keywords Linear elasticity $\cdot$ shell $\cdot$ multi structure

PACS PACS 4615Cc $\cdot$ PACS 4670De

Mathematics Subject Classification (2000) MSC 74K30 - MSC 74G99

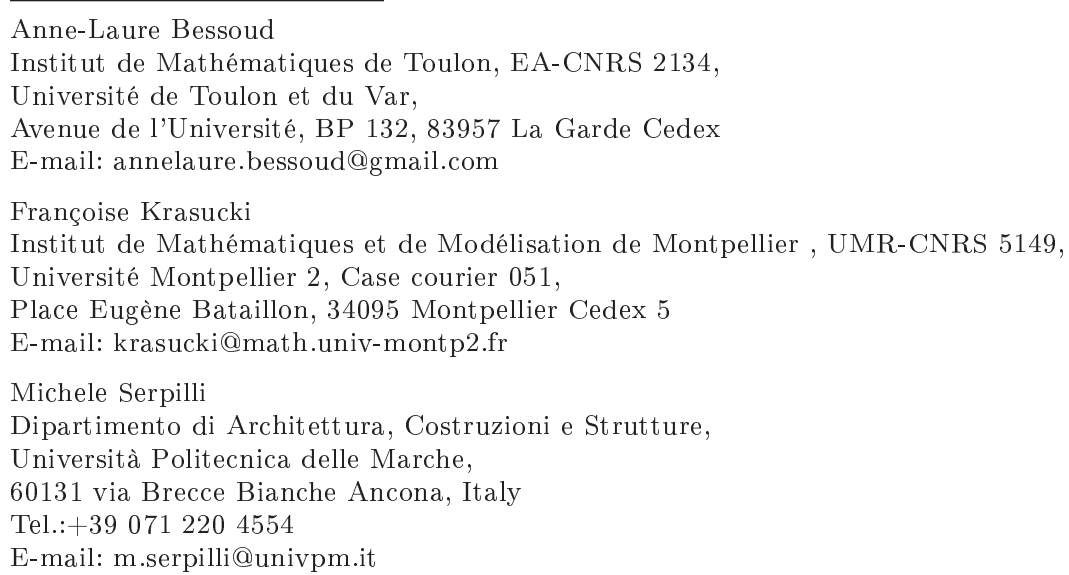




\section{Introduction}

The modeling of complex structures obtained assembling simpler elements with very different geometric and/or material characteristics is a source of a variety of problems of practical importance. The successful application of the asymptotic methods to obtain a mathematical justification of the most used models of plates and shells has stimulated the research toward a rational simplification of the modeling of complex structures obtained joining elements of different dimensions and/or materials of highly contrasted properties. The first modeling of junctions between elements of different dimension is due to [6], [9]. The thin inclusion of a third material between two other ones denoted $\Omega^{+}$and $\Omega^{-}$when the rigidity properties of the inclusion are highly contrasted with respect to those of the surrounding materials has also been deeply investigated and one can refer without claim of completeness to [11, $3,4,8]$. New motivations appear in [7], where the authors, in order to justify some methods used in the FEM approximation, have studied the asymptotic behavior of a shell-like inclusion of $\frac{1}{\varepsilon^{p}}$-rigidity $(p=1$ or $p=3)$ in a threedimensional domain using a Naghdi linear shell model [5], [10]. In a slightly different geometrical and mechanical context, Bessoud et al. [2] have studied the behavior of a $\varepsilon$-thin three-dimensional layer of $\frac{1}{\varepsilon}$-rigidity. More precisely, they assume that the thin layer can be written as $\omega \times]-\varepsilon, \varepsilon[$ where $\omega$ is a projectable two-dimensional surface, and that all the materials are linearly elastic anisotropic. Then the limit problem is a Ventcel-type transmission problem between two three-dimensional linearly elastic anisotropic bodies $\Omega^{+}$and $\Omega^{-}$ on their common boundary $\omega$. When $\omega$ is planar and in the isotropic case, the associated surface energy term can be interpreted as the membranal energy of a Kirchhoff-Love plate.

In the present paper, we study the situation where the shell-like thin layer is obtained by the translation along the normal direction of a general twodimensional surface. Using a system of curvilinear coordinates we deduce the formal limit problem for the two cases $p=1$ and $p=3$. When $p=1$ we find in section 5 that the thin layer behaves as a membrane shell and in section 6 when $p=3$ it behaves as a flexural shell. In this way we recover the limit problems analogous to those of [7] where the authors a priori assume a shell-like energy in the thin layer. The formal limit problems so obtained are justified in section 8 by proving strong convergence results in a suitable functional framework using the Korn type results of section 7 . Let us remark that in our asymptotic approach thanks to our choice of the boundary conditions, there is no need to take care of the space of inextensional or pure bending displacements of $\omega$ as in [7] and in the usual asymptotic analysis of shell models by themselves (see e. g; $[5],[1],[12]$ and the references therein). Indeed the limit shell behaviour of $\omega$ is taken into account only in unusual transmission conditions and so completely controlled by the surrounding bodies. These unusual transmission conditions imply that the global displacement of the assembly is continuous across the interface $\omega$. The difference between the case $p=1$ and $p=3$ appears in the interface jump stress conditions. 


\section{Geometrical preliminaries}

\subsection{Three-dimensional curvilinear coordinates}

This section is aimed at laying down an appropriate ground for the rest of the article. In the sequel, Greek indices range in the set $\{1,2\}$, Latin indices range in the set $\{1,2,3\}$, and the summation convention with respect to the repeated indices is adopted.

Let us consider a three-dimensional Euclidian space identified by $\mathbb{R}^{3}$ and such that the three vectors $\mathbf{e}_{i}$ form an orthonormal basis. Let $\Omega$ be a nonempty open subset of $\mathbb{R}^{3}$. A mapping $\Theta \in \mathcal{C}^{3}\left(\Omega ; \mathbb{R}^{3}\right)$ is an immersion if the three vectors $\partial_{i} \Theta(x)$ are linearly independent for all $x=\left(x_{i}\right) \in \Omega$. The image $\Theta(\Omega)$ is always an open set immersed in $\mathbb{R}^{3}$. The three coordinates $x_{i}$ of a point $x \in \Omega$ represent the curvilinear coordinates of the point $\boldsymbol{\Theta}(x) \in \boldsymbol{\Theta}(\Omega)$, while the three coordinates $\Theta_{i}(x)$ of the point $\boldsymbol{\Theta}(x) \in \boldsymbol{\Theta}(\Omega)$ are the Cartesian coordinates.

The three vectors $\mathbf{g}_{i}(x):=\partial_{i} \boldsymbol{\Theta}(x)$ form the covariant basis at $\boldsymbol{\Theta}(x)$ and the three vectors $\mathbf{g}^{j}(x)$, defined by the nine independent relations $\mathbf{g}_{i}(x) \cdot \mathbf{g}^{j}(x)=$ $\delta_{i}^{j}$ for all $x \in \Omega,\left(\delta_{i}^{j}\right.$ denotes the Kronecker symbol) form the contravariant basis at $\boldsymbol{\Theta}(x)$. The immersion $\Theta$ induces a Riemannian metric on $\Omega$, defined respectively by its covariant components $g_{i j}(x):=\mathbf{g}_{i}(x) \cdot \mathbf{g}_{j}(x)$, and contravariant components $g^{k \ell}(x):=\mathbf{g}^{k}(x) \cdot \mathbf{g}^{\ell}(x)$. The contravariant components of this metric can be analogously defined by $\left(g^{k \ell}(x)\right)=\left(g_{i j}(x)\right)^{-1}$ for all $x \in \Omega$.

This metric induces a Levi-Civita connection in the manifold $\Omega$ defined by the Christoffel symbols of the second kind $\Gamma_{i j}^{p}:=\mathbf{g}^{p} \cdot \partial_{i} \mathbf{g}_{j}=\Gamma_{j i}^{p}$.

Let there be given a vector field defined over $\boldsymbol{\Theta}(\Omega)$. We can rewrite this vector field as a linear combination $\mathbf{v}=v_{i} \mathbf{g}^{i}$ of the vector fields $\mathbf{g}^{i}: \Omega \rightarrow \mathbb{R}^{3}$, where $v_{i}=\mathbf{v} \cdot \mathbf{g}_{i}$ are the covariant components of the vector field $\mathbf{v}$. The covariant derivatives $v_{i \| j} \in \mathcal{C}^{0}(\Omega)$ of the covariant components $v_{i} \in \mathcal{C}^{1}(\Omega)$ are defined by $v_{i \| j}:=\partial_{j} v_{i}-\Gamma_{i j}^{p} v_{p}$. The covariant derivatives $T^{i j} \|_{k} \in \mathcal{C}^{0}(\Omega)$ of the second-order tensor field with contravariant components $T^{i j} \in \mathcal{C}^{1}(\Omega)$ are defined by $T^{i j} \|_{k}:=\partial_{k} T^{i j}+\Gamma_{\ell j}^{i} T^{\ell k}+\Gamma_{\ell k}^{j} T^{\ell i}$.

With every displacement field $\mathbf{v}$, we associate the linearized change of metric tensor defined as follows:

$$
e_{i j}(\mathbf{v}):=\frac{1}{2}\left(v_{i \| j}+v_{j \| i}\right)
$$

\subsection{Curvilinear coordinates on a surface}

Let $\omega$ be a non-empty open subset in $\mathbb{R}^{2}$. The coordinates of $\widetilde{x} \in \omega$ are denoted by $x_{\alpha}$. A mapping $\boldsymbol{\theta} \in \mathcal{C}^{3}\left(\omega ; \mathbb{R}^{3}\right)$ is an immersion if the two vectors $\partial_{\alpha} \boldsymbol{\theta}(\widetilde{x})$ are linearly independent at each point $\widetilde{x}=\left(x_{\alpha}\right) \in \omega$. The image $S:=\boldsymbol{\theta}(\omega)$ is a surface immersed in $\mathbb{R}^{3}$, equipped with $x_{\alpha}$ curvilinear coordinates.

The two vectors $\mathbf{a}_{\alpha}(\widetilde{x}):=\partial_{\alpha} \boldsymbol{\theta}(\widetilde{x})$ form the covariant basis of the tangent plane to the surface $S$ at $\boldsymbol{\theta}(\widetilde{x})$, and the two vectors $\mathbf{a}^{\beta}(\widetilde{x})$ defined by the 
relations $\mathbf{a}_{\alpha}(\widetilde{x}) \cdot \mathbf{a}^{\beta}(\widetilde{x})=\delta_{\alpha}^{\beta}$, form the contravariant basis of the tangent plane to the surface $S$ at $\boldsymbol{\theta}(\widetilde{x})$. The unit normal vector to $S$ at $\boldsymbol{\theta}(\widetilde{x})$ is defined by $\mathbf{a}_{3}(\widetilde{x})=\mathbf{a}^{3}(\widetilde{x}):=\frac{\mathbf{a}_{1}(\widetilde{x}) \wedge \mathbf{a}_{2}(\widetilde{x})}{\left|\mathbf{a}_{1}(\widetilde{x}) \wedge \mathbf{a}_{2}(\widetilde{x})\right|}$.

The covariant components of the first fundamental form of the surface are defined by $a_{\alpha \beta}(\widetilde{x}):=\mathbf{a}_{\alpha}(\widetilde{x}) \cdot \mathbf{a}_{\beta}(\widetilde{x})$, and its contravariant components are defined by $a^{\alpha \beta}(\widetilde{x}):=\mathbf{a}^{\alpha}(\widetilde{x}) \cdot \mathbf{a}^{\beta}(\widetilde{x})$.

The covariant components of the second fundamental form of the surface are defined by $b_{\alpha \beta}(\widetilde{x}):=\partial_{\alpha} \mathbf{a}_{\beta}(\widetilde{x}) \cdot \mathbf{a}_{3}(\widetilde{x})$, and its mixed components are defined by $b_{\alpha}^{\tau}(\widetilde{x}):=a^{\tau \beta}(\widetilde{x}) b_{\alpha \beta}(\widetilde{x})$.

The Christoffel symbols on the surface $S$ of the second kind are given by $\Gamma_{\alpha \beta}^{\tau}(\widetilde{x}):=\mathbf{a}^{\tau}(\widetilde{x}) \cdot \partial_{\alpha} \mathbf{a}_{\beta}(\widetilde{x})$.

Any vector field on a surface can be written as a linear combination $\boldsymbol{\eta}=$ $\eta_{i} \mathbf{a}^{i}$ of the vector field $\mathbf{a}^{i}: \omega \rightarrow \mathbb{R}^{3}$, where the functions $\eta_{i}=\boldsymbol{\eta} \cdot \mathbf{a}_{i}$ are the covariant components of the vector field $\boldsymbol{\eta}$. The covariant derivatives $\eta_{\alpha \mid \beta} \in$ $\mathcal{C}^{0}(\omega)$ of the covariant components $\eta_{\alpha} \in \mathcal{C}^{1}(\omega)$ are defined by $\eta_{\alpha \mid \beta}:=\partial_{\beta} \eta_{\alpha}-$ $\Gamma_{\alpha \beta}^{\tau} \eta_{\tau}$. The covariant derivatives $\left.T^{\alpha \beta}\right|_{\tau} \in \mathcal{C}^{0}(\omega)$ of the second-order tensor field with contravariant components $T^{\alpha \beta} \in \mathcal{C}^{1}(\omega)$ are defined by $\left.T^{\alpha \beta}\right|_{\tau}:=\partial_{\tau} T^{\alpha \beta}+$ $\Gamma_{\sigma \tau}^{\alpha} T^{\beta \sigma}+\Gamma_{\tau \sigma}^{\beta} T^{\alpha \sigma}$. The covariant derivatives of the curvature tensor defined by means of its mixed components are defined by $\left.b_{\beta}^{\tau}\right|_{\alpha}:=\partial_{\alpha} b_{\beta}^{\tau}+\Gamma_{\alpha \sigma}^{\tau} b_{\beta}^{\sigma}-\Gamma_{\alpha \beta}^{\sigma} b_{\sigma}^{\tau}$. For more details about differential geometry of surfaces, see e. g. [5].

With every displacement field $\boldsymbol{\eta}$, we associate the linearized change of metric tensor field defined by

$$
\gamma_{\alpha \beta}(\boldsymbol{\eta}):=\frac{1}{2}\left(\partial_{\beta} \eta_{\alpha}+\partial_{\alpha} \eta_{\beta}\right)-\Gamma_{\alpha \beta}^{\sigma} \eta_{\sigma}-b_{\alpha \beta} \eta_{3}
$$

and the linearized change of curvature tensor field, defined by

$$
\begin{aligned}
\rho_{\alpha \beta}(\boldsymbol{\eta}):= & \partial_{\alpha \beta} \eta_{3}-\Gamma_{\alpha \beta}^{\sigma} \partial_{\sigma} \eta_{3}-b_{\alpha}^{\sigma} b_{\sigma \beta} \eta_{3}+b_{\alpha}^{\sigma}\left(\partial_{\beta} \eta_{\sigma}-\Gamma_{\beta \sigma}^{\tau} \eta_{\tau}\right)+ \\
& +b_{\beta}^{\tau}\left(\partial_{\alpha} \eta_{\tau}-\Gamma_{\alpha \tau}^{\sigma} \eta_{\sigma}\right)+\left(\partial_{\alpha} b_{\beta}^{\tau}+\Gamma_{\alpha \sigma}^{\tau} b_{\beta}^{\sigma}-\Gamma_{\alpha \beta}^{\sigma} b_{\sigma}^{\tau}\right) \eta_{\tau}
\end{aligned}
$$

The symmetric tensor fields $\left(\gamma_{\alpha \beta}\right)$ and $\left(\rho_{\alpha \beta}\right)$ play a key role in the theory of linearly elastic shells (see, e.g., P.G. Ciarlet [5]).

\section{Position of the problem}

Let $\Omega^{+}$and $\Omega^{-}$be two disjoint open domains with smooth boundaries $\partial \Omega^{+}$ and $\partial \Omega^{-}$. Let $\omega:=\left\{\partial \Omega^{+} \cap \partial \Omega^{-}\right\}^{\circ}$ be the interior of the common part of the boundaries which is assumed to be a non empty domain in $\mathbb{R}^{2}$ having a positive two-dimensional measure and let $\boldsymbol{\theta} \in \mathcal{C}^{2}\left(\bar{\omega} ; \mathbb{R}^{3}\right)$ be an immersion.

Let $0<\varepsilon<1$ be an adimensional small real parameter. Let us consider $\left.\Omega^{m, \varepsilon}:=\omega \times\right]-\varepsilon, \varepsilon\left[\right.$ and $S^{ \pm, \varepsilon}:=\omega \times\{ \pm \varepsilon\}$. Let $x^{\varepsilon}$ denote the generic point in the set $\bar{\Omega}^{m, \varepsilon}$ with $x_{\alpha}^{\varepsilon}=x_{\alpha}$. We consider a shell-like domain with middle surface $\boldsymbol{\theta}(\bar{\omega})$ and thickness $2 \varepsilon$, whose reference configuration is the image $\boldsymbol{\Theta}^{m, \varepsilon}\left(\bar{\Omega}^{m, \varepsilon}\right) \subset \mathbb{R}^{3}$ of the set $\bar{\Omega}^{m, \varepsilon}$ through the mapping given by

$$
\boldsymbol{\Theta}^{m, \varepsilon}\left(x^{\varepsilon}\right):=\boldsymbol{\theta}(\widetilde{x})+x_{3}^{\varepsilon} \mathbf{a}_{3}(\widetilde{x}) \text {, for all } x^{\varepsilon}=\left(\widetilde{x}, x_{3}^{\varepsilon}\right) \in \bar{\Omega}^{m, \varepsilon} \text {. }
$$




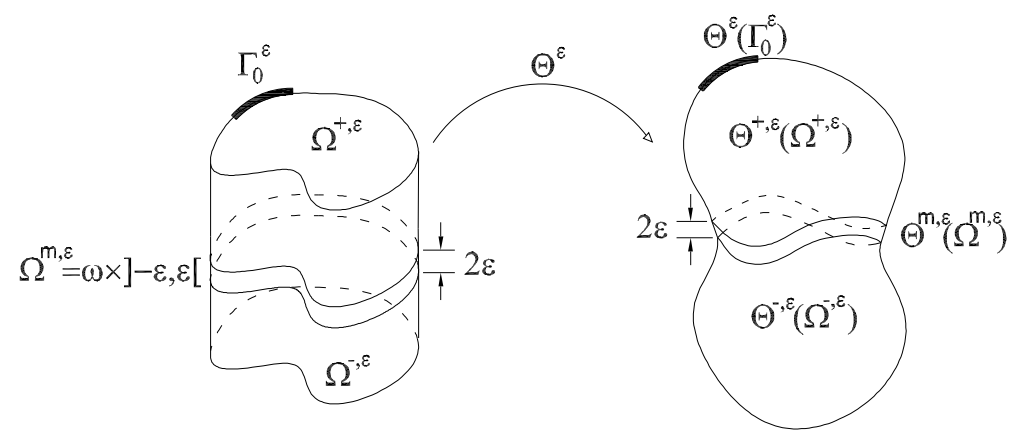

Fig. 1 Initial and reference configuration of the assembly

We denote by $\Omega^{+, \varepsilon}$ (resp. $\Omega^{-, \varepsilon}$ ) the translation of $\Omega^{+}$(resp $\Omega^{-}$) in the direction $\mathbf{e}_{3}$ (resp.--e 3 ) of the quantity $\varepsilon$ and we set $\Omega^{\varepsilon}=\Omega^{-, \varepsilon} \cup S^{-, \varepsilon} \cup \Omega^{m, \varepsilon} \cup$ $S^{+, \varepsilon} \cup \Omega^{+, \varepsilon}$.

Moreover, we suppose that there exists an immersion $\Theta^{\varepsilon}: \bar{\Omega}^{\varepsilon} \rightarrow \mathbb{R}^{3}$ defined as follows:

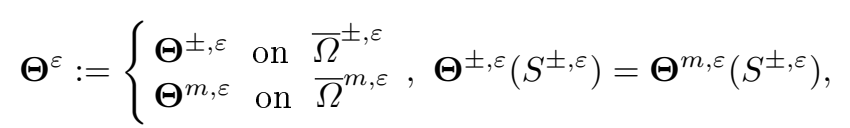

where $\Theta^{ \pm, \varepsilon}: \bar{\Omega}^{ \pm, \varepsilon} \rightarrow \mathbb{R}^{3}$ are immersions over $\bar{\Omega}^{ \pm, \varepsilon}$ defining the curvilinear coordinates on $\bar{\Omega}^{ \pm, \varepsilon}$. Let us stress that the physical domain of the assembly is obtained by inserting in the direction $\mathbf{a}_{3}$ the shell within the two bodies, see Fig. 1. The structure is clamped on $\Gamma_{0}^{\varepsilon}$ and the complementary part of the boundary is free. Obviously we can consider other type of boundary conditions. The structure is also submitted to applied body forces $f_{i}^{\varepsilon}$ so that the work of the external loading is given by the linear form

$$
L^{\varepsilon}\left(\mathbf{v}^{\varepsilon}\right):=\int_{\Omega^{ \pm, \varepsilon}} f_{i}^{\varepsilon} v_{i}^{\varepsilon} d x^{\varepsilon}
$$

We suppose that the materials are linearly elastic and isotropic with Lamé's constants $\lambda^{ \pm, \varepsilon}$ and $\mu^{ \pm, \varepsilon}$ for $\Omega^{ \pm, \varepsilon}, \lambda^{m, \varepsilon}$ and $\mu^{m, \varepsilon}$ for $\Omega^{m, \varepsilon}$. As usual we assume that $3 \lambda^{ \pm, \varepsilon}+2 \mu^{ \pm, \varepsilon}>0, \mu^{ \pm, \varepsilon}>0,3 \lambda^{m, \varepsilon}+2 \mu^{m, \varepsilon}>0, \mu^{m, \varepsilon}>0$.

The physical variational problem in curvilinear coordinates defined over the variable domain $\Omega^{\varepsilon}$ can be written as

$$
\left\{\begin{array}{l}
\text { Find } \mathbf{u}^{\varepsilon} \in V^{\varepsilon}:=\left\{\mathbf{v}^{\varepsilon} \in H^{1}\left(\Omega^{\varepsilon} ; \mathbb{R}^{3}\right) ; \mathbf{v}_{\mid \Gamma_{0}^{\varepsilon}}^{\varepsilon}=\mathbf{0}\right\} \quad \text { such that } \\
A^{-, \varepsilon}\left(\mathbf{u}^{\varepsilon}, \mathbf{v}^{\varepsilon}\right)+A^{+, \varepsilon}\left(\mathbf{u}^{\varepsilon}, \mathbf{v}^{\varepsilon}\right)+A^{m, \varepsilon}\left(\mathbf{u}^{\varepsilon}, \mathbf{v}^{\varepsilon}\right)=L^{\varepsilon}\left(\mathbf{v}^{\varepsilon}\right) \text { for all } \mathbf{v}^{\varepsilon} \in V^{\varepsilon}
\end{array}\right.
$$


The bilinear forms $A^{ \pm, \varepsilon}(\cdot, \cdot)$ and $A^{m, \varepsilon}(\cdot, \cdot)$ are defined by

$$
\begin{aligned}
& A^{ \pm, \varepsilon}\left(\mathbf{u}^{\varepsilon}, \mathbf{v}^{\varepsilon}\right):=\int_{\Omega^{ \pm, \varepsilon}} A_{ \pm}^{i j k \ell, \varepsilon} e_{k \ell}^{\varepsilon}\left(\mathbf{u}^{\varepsilon}\right) e_{i j}^{\varepsilon}\left(\mathbf{v}^{\varepsilon}\right) \sqrt{g^{ \pm, \varepsilon}} d x^{\varepsilon} \\
& A^{m, \varepsilon}\left(\mathbf{u}^{\varepsilon}, \mathbf{v}^{\varepsilon}\right):=\int_{\Omega^{m, \varepsilon}} A_{m}^{i j k \ell, \varepsilon} e_{k \ell}^{\varepsilon}\left(\mathbf{u}^{\varepsilon}\right) e_{i j}^{\varepsilon}\left(\mathbf{v}^{\varepsilon}\right) \sqrt{g^{m, \varepsilon}} d x^{\varepsilon}
\end{aligned}
$$

Here, $A^{i j k \ell, \varepsilon}:=\lambda^{\varepsilon} g^{i j, \varepsilon} g^{k \ell, \varepsilon}+\mu^{\varepsilon}\left(g^{i k, \varepsilon} g^{j \ell, \varepsilon}+g^{i \ell, \varepsilon} g^{j k, \varepsilon}\right)$ are the contravariant components of the elasticity tensor and $g^{\varepsilon}:=\operatorname{det}\left(g_{i j}^{\varepsilon}\right)$.

If we suppose that $f_{i}^{\varepsilon} \in L^{2}\left(\Omega^{ \pm, \varepsilon}\right)$, then Lax-Milgram's lemma ensures existence of a unique solution for problem (1).

In order to study the asymptotic behavior of the solution of problem (1) when $\varepsilon$ tends to zero, we rewrite the problem on a fixed domain $\Omega$ independent of $\varepsilon$. By using the approach of [5], we consider the bijection $\pi^{\varepsilon}: x \in \bar{\Omega} \mapsto x^{\varepsilon} \in$ $\bar{\Omega}^{\varepsilon}$ given by

$$
\begin{cases}\pi^{\varepsilon}\left(x_{1}, x_{2}, x_{3}\right)=\left(x_{1}, x_{2}, x_{3}-(1-\varepsilon)\right), & \text { for all } x \in \bar{\Omega}_{t r}^{+}, \\ \pi^{\varepsilon}\left(x_{1}, x_{2}, x_{3}\right)=\left(x_{1}, x_{2}, \varepsilon x_{3}\right), & \text { for all } x \in \bar{\Omega}^{m}, \\ \pi^{\varepsilon}\left(x_{1}, x_{2}, x_{3}\right)=\left(x_{1}, x_{2}, x_{3}+(1-\varepsilon)\right), & \text { for all } x \in \bar{\Omega}_{t r}^{-},\end{cases}
$$

where $\left.\Omega_{t r}^{ \pm}:=\left\{x \pm \mathbf{e}_{3}, x \in \Omega^{ \pm}\right\}, \Omega^{m}:=\omega \times\right]-1,1\left[\right.$ and $S^{ \pm}:=\omega \times\{ \pm 1\}$. In order to simplify the notation, we still denote by $\Omega^{ \pm}$the set $\Omega_{t r}^{ \pm}$, and set $\Omega=\Omega^{-} \cup S^{-} \cup \Omega^{m} \cup S^{+} \cup \Omega^{+}$. Consequently, one has $\partial_{\alpha}^{\varepsilon}=\partial_{\alpha}$ and $\partial_{3}^{\varepsilon}=$ $\frac{1}{\varepsilon} \partial_{3}$ in $\Omega^{m}$.

With the unknowns $\mathbf{u}^{\varepsilon}$ and the test functions $\mathbf{v}^{\varepsilon}$ appearing in formulation (1), we associate respectively the scaled unknowns $\mathbf{u}$ and the scaled test functions $\mathbf{v}$ transformed by $\pi^{\varepsilon}$ by means of the following relations:

$$
\begin{aligned}
& \mathbf{u}(\varepsilon)(x):=\mathbf{u}^{\varepsilon}\left(x^{\varepsilon}\right), \text { for all } x^{\varepsilon}=\pi^{\varepsilon}(x) \in \bar{\Omega}^{\varepsilon}, \\
& \mathbf{v}(x):=\mathbf{v}^{\varepsilon}\left(x^{\varepsilon}\right), \quad \text { for all } x^{\varepsilon}=\pi^{\varepsilon}(x) \in \bar{\Omega}^{\varepsilon} .
\end{aligned}
$$

For $\varepsilon$ sufficiently small, we associate with the functions $A_{ \pm}^{i j k \ell, \varepsilon}, g^{ \pm, \varepsilon}, \Gamma_{i j}^{p, \varepsilon}$ : $\bar{\Omega}^{ \pm, \varepsilon} \rightarrow \mathbb{R}$ the functions $A_{ \pm}^{i j k \ell}, g^{ \pm}, \Gamma_{i j}^{p}: \bar{\Omega}^{ \pm} \rightarrow \mathbb{R}$ defined by

$$
\begin{array}{ll}
A_{ \pm}^{i j k \ell}(x):=A_{ \pm}^{i j k \ell, \varepsilon}\left(x^{\varepsilon}\right), \text { for all } x^{\varepsilon}=\pi^{\varepsilon}(x) \in \bar{\Omega}^{ \pm, \varepsilon} \\
g^{ \pm}(x):=g^{ \pm, \varepsilon}\left(x^{\varepsilon}\right), & \text { for all } x^{\varepsilon}=\pi^{\varepsilon}(x) \in \bar{\Omega}^{ \pm, \varepsilon}, \\
\Gamma_{i j}^{p}(x):=\Gamma_{i j}^{p, \varepsilon}\left(x^{\varepsilon}\right), & \text { for all } x^{\varepsilon}=\pi^{\varepsilon}(x) \in \bar{\Omega}^{ \pm, \varepsilon}
\end{array}
$$

and we associate with the functions $A_{m}^{i j k \ell, \varepsilon}, g^{m, \varepsilon}, \Gamma_{i j}^{p, \varepsilon}: \bar{\Omega}^{m, \varepsilon} \rightarrow \mathbb{R}$ the functions $A_{m}^{i j k \ell}(\varepsilon), g^{m}(\varepsilon), \Gamma_{i j}^{p}(\varepsilon): \bar{\Omega}^{m} \rightarrow \mathbb{R}$ defined by

$$
\begin{array}{ll}
A_{m}^{i j k \ell}(\varepsilon)(x):=A_{m}^{i j k \ell, \varepsilon}\left(x^{\varepsilon}\right), & \text { for all } x^{\varepsilon}=\pi^{\varepsilon}(x) \in \bar{\Omega}^{m, \varepsilon}, \\
g^{m}(\varepsilon)(x):=g^{m, \varepsilon}\left(x^{\varepsilon}\right), & \text { for all } x^{\varepsilon}=\pi^{\varepsilon}(x) \in \bar{\Omega}^{m, \varepsilon} \\
\Gamma_{i j}^{p}(\varepsilon)(x):=\Gamma_{i j}^{p, \varepsilon}\left(x^{\varepsilon}\right), & \text { for all } x^{\varepsilon}=\pi^{\varepsilon}(x) \in \bar{\Omega}^{m, \varepsilon} .
\end{array}
$$


We assume that the rigidity of the shell-like layer has order of magnitude $\frac{1}{\varepsilon^{p}}$ with $p \geq 1$. From the previous assumptions it then follows that:

$$
\left\{\begin{array}{l}
A_{m}^{\alpha \beta \sigma 3}(\varepsilon)=A_{m}^{\alpha 333}(\varepsilon)=0 \\
A_{m}^{i j k \ell}(\varepsilon) \sqrt{g^{m}(\varepsilon)}= \\
\quad=\frac{1}{\varepsilon^{p}} A_{m}^{i j k \ell}(0) \sqrt{a}+\frac{1}{\varepsilon^{p-1}} B_{m}^{i j k \ell, 1}+\frac{1}{\varepsilon^{p-2}} B_{m}^{i j k \ell, 2}+O\left(\varepsilon^{2-p}\right),
\end{array}\right.
$$

where $a:=\operatorname{det}\left(a_{\alpha \beta}\right)$,

$$
\begin{gathered}
A_{m}^{\alpha \beta \sigma \tau}(0):=\lambda^{m} a^{\alpha \beta} a^{\sigma \tau}+\mu^{m}\left(a^{\alpha \sigma} a^{\beta \tau}+a^{\alpha \tau} a^{\beta \sigma}\right), \\
A_{m}^{\alpha \beta 33}(0):=\lambda^{m} a^{\alpha \beta}, \quad A_{m}^{\alpha 3 \sigma 3}(0):=\mu^{m} a^{\alpha \sigma}, \quad A_{m}^{3333}(0):=\lambda^{m}+2 \mu^{m},
\end{gathered}
$$

and the order symbol $O\left(\varepsilon^{2-p}\right)$ is meant with respect to the norm of $\mathcal{C}^{0}\left(\overline{\Omega^{m}}\right)$.

The covariant components of the linearized change of the metric tensor $e_{i j}(\varepsilon ; \mathbf{v}) \in L^{2}\left(\Omega^{m}\right)$, transformed by $\pi^{\varepsilon}$ and associated with the displacement field $\mathbf{v} \in H^{1}\left(\Omega^{m} ; \mathbb{R}^{3}\right)$, are defined as follows:

$$
\begin{aligned}
e_{\alpha \beta}(\varepsilon ; \mathbf{v}):= & \frac{1}{2}\left(\partial_{\beta} v_{\alpha}+\partial_{\alpha} v_{\beta}\right)-\Gamma_{\alpha \beta}^{p}(\varepsilon) v_{p} \\
e_{\alpha 3}(\varepsilon ; \mathbf{v}):= & \frac{1}{2}\left(\frac{1}{\varepsilon} \partial_{3} v_{\alpha}+\partial_{\alpha} v_{3}\right)-\Gamma_{\alpha 3}^{\sigma}(\varepsilon) v_{\sigma} \\
& e_{33}(\varepsilon ; \mathbf{v}):=\frac{1}{\varepsilon} \partial_{3} v_{3} .
\end{aligned}
$$

As in [5] one can prove that in $\Omega^{m}$ the functions $\Gamma_{i j}^{p}(\varepsilon)$ satisfy :

$$
\left\{\begin{array}{l}
\Gamma_{\alpha \beta}^{\sigma}(\varepsilon)=\Gamma_{\alpha \beta}^{\sigma}-\left.\varepsilon x_{3} b_{\beta}^{\sigma}\right|_{\alpha}+O\left(\varepsilon^{2}\right) \\
\Gamma_{\alpha \beta}^{3}(\varepsilon)=b_{\alpha \beta}-\varepsilon x_{3} b_{\alpha}^{\sigma} b_{\sigma \beta} \\
\Gamma_{\alpha 3}^{\sigma}(\varepsilon)=-b_{\alpha}^{\sigma}-\varepsilon x_{3} b_{\alpha}^{\tau} b_{\tau}^{\sigma}+O\left(\varepsilon^{2}\right) \\
\Gamma_{\alpha 3}^{3}(\varepsilon)=\Gamma_{33}^{p}(\varepsilon)=0
\end{array}\right.
$$

where the order symbols $O(\varepsilon)$ and $O\left(\varepsilon^{2}\right)$ are meant with respect to the norm of $\mathcal{C}^{0}\left(\overline{\Omega^{m}}\right)$.

For later use we define $\gamma_{\alpha \beta}(\mathbf{v})$ and $\rho_{\alpha \beta}(\mathbf{v})$ in $\Omega^{m}$ with the same formulae employed in section 2.2 for the surface $\omega$ :

$$
\begin{gathered}
\gamma_{\alpha \beta}(\mathbf{v}):=\frac{1}{2}\left(\partial_{\beta} v_{\alpha}+\partial_{\alpha} v_{\beta}\right)-\Gamma_{\alpha \beta}^{\sigma} v_{\sigma}-b_{\alpha \beta} v_{3}, \\
\rho_{\alpha \beta}(\mathbf{v}):=\begin{array}{c}
\partial_{\alpha \beta} v_{3}-\Gamma_{\alpha \beta}^{\sigma} \partial_{\sigma} v_{3}-b_{\alpha}^{\sigma} b_{\sigma \beta} v_{3}+b_{\alpha}^{\sigma}\left(\partial_{\beta} v_{\sigma}-\Gamma_{\beta \sigma}^{\tau} v_{\tau}\right)+ \\
+b_{\beta}^{\tau}\left(\partial_{\alpha} v_{\tau}-\Gamma_{\alpha \tau}^{\sigma} v_{\sigma}\right)+\left(\partial_{\alpha} b_{\beta}^{\tau}+\Gamma_{\alpha \sigma}^{\tau} b_{\beta}^{\sigma}-\Gamma_{\alpha \beta}^{\sigma} b_{\sigma}^{\tau}\right) v_{\tau} .
\end{array}
\end{gathered}
$$

For simplicity we assumed that the shell-like inclusion is free of charges, then it follows that $L^{\varepsilon}\left(\mathbf{v}^{\varepsilon}\right)=L(\mathbf{v})$. According to the previous assumptions, problem 
(1) can be reformulated on the fixed domain $\Omega$ independent of $\varepsilon$. We obtain the following scaled problem:

$$
\left\{\begin{array}{l}
\text { Find } \mathbf{u}(\varepsilon) \in V:=\left\{\mathbf{v} \in H^{1}\left(\Omega ; \mathbb{R}^{3}\right) ; \quad \mathbf{v}_{\mid \Gamma_{0}}=\mathbf{0}\right\} \quad \text { such that } \\
A^{-}(\mathbf{u}(\varepsilon), \mathbf{v})+A^{+}(\mathbf{u}(\varepsilon), \mathbf{v})+\varepsilon A^{m}(\mathbf{u}(\varepsilon), \mathbf{v})=L(\mathbf{v}) \text { for all } \mathbf{v} \in V
\end{array}\right.
$$

where

$$
\begin{gathered}
A^{ \pm}(\mathbf{u}(\varepsilon), \mathbf{v}):=\int_{\Omega^{ \pm}} A_{ \pm}^{i j k \ell} e_{k \ell}(\mathbf{u}(\varepsilon)) e_{i j}(\mathbf{v}) \sqrt{g^{ \pm}} d x \\
A^{m}(\mathbf{u}(\varepsilon), \mathbf{v}):=\int_{\Omega^{m}} A_{m}^{i j k \ell}(\varepsilon) e_{k \ell}(\varepsilon ; \mathbf{u}(\varepsilon)) e_{i j}(\varepsilon ; \mathbf{v}) \sqrt{g^{m}(\varepsilon)} d x .
\end{gathered}
$$

In the sequel, only if necessary, we denote by $\mathbf{v}^{ \pm}$, resp $\mathbf{v}^{m}$, the restriction of the function $\mathbf{v}$ to $\Omega^{ \pm}$, resp $\Omega^{m}$.

\section{Asymptotic expansion}

We can now perform an asymptotic analysis of the rescaled problem (6). We distinguish the two cases when the rigidity of the shell-like layer has its order of magnitude equal to $\frac{1}{\varepsilon}$ or $\frac{1}{\varepsilon^{3}}$ with respect to the rigidities of the surrounding three-dimensional bodies.

Since the rescaled problem (6) has a polynomial structure with respect to the small parameter $\varepsilon$, we can look for a formal development of the solution:

$$
\mathbf{u}(\varepsilon)=\mathbf{u}^{0}+\varepsilon \mathbf{u}^{1}+\varepsilon^{2} \mathbf{u}^{2}+\ldots,
$$

with $\mathbf{u}^{q} \in V, q \in \mathbb{N}$.

The above formal asymptotic expansion of the scaled unknowns and the asymptotic behavior of functions $\Gamma_{i j}^{p}(\varepsilon)$ induce the formal asymptotic expansion for the scaled linearized strains in $\Omega^{m}$ of the form:

$$
e_{i j}(\varepsilon)=\frac{1}{\varepsilon} e_{i j}^{-1}+e_{i j}^{0}+\varepsilon e_{i j}^{1}+\varepsilon^{2} e_{i j}^{2}+\ldots,
$$

where

$$
\begin{gathered}
\left\{\begin{array} { l } 
{ e _ { \alpha \beta } ^ { - 1 } : = 0 , } \\
{ e _ { \alpha 3 } ^ { - 1 } : = \frac { 1 } { 2 } \partial _ { 3 } u _ { \alpha } ^ { 0 } , } \\
{ e _ { 3 3 } ^ { - 1 } : = \partial _ { 3 } u _ { 3 } ^ { 0 } , }
\end{array} \quad \left\{\begin{array}{l}
e_{\alpha \beta}^{0}:=\frac{1}{2}\left(\partial_{\beta} u_{\alpha}^{0}+\partial_{\alpha} u_{\beta}^{0}\right)-\Gamma_{\alpha \beta}^{\sigma} u_{\sigma}^{0}-b_{\alpha \beta} u_{3}^{0}, \\
e_{\alpha 3}^{0}:=\frac{1}{2}\left(\partial_{3} u_{\alpha}^{1}+\partial_{\alpha} u_{3}^{0}\right)+b_{\alpha}^{\sigma} u_{\sigma}^{0}, \\
e_{33}^{0}:=\partial_{3} u_{3}^{1},
\end{array}\right.\right. \\
\left\{\begin{array}{l}
e_{\alpha \beta}^{1}:=\frac{1}{2}\left(\partial_{\beta} u_{\alpha}^{1}+\partial_{\alpha} u_{\beta}^{1}\right)-\Gamma_{\alpha \beta}^{\sigma} u_{\sigma}^{1}-b_{\alpha \beta} u_{3}^{1}+x_{3}\left(\left.b_{\beta}^{\sigma}\right|_{\alpha} u_{\sigma}^{0}+b_{\alpha}^{\sigma} b_{\sigma \beta} u_{3}^{0}\right), \\
e_{\alpha 3}^{1}:=\frac{1}{2}\left(\partial_{3} u_{\alpha}^{2}+\partial_{\alpha} u_{3}^{1}\right)+b_{\alpha}^{\sigma} u_{\sigma}^{1}+x_{3} b_{\alpha}^{\tau} b_{\tau}^{\sigma} u_{\sigma}^{0}, \\
e_{33}^{1}:=\partial_{3} u_{3}^{2} .
\end{array}\right.
\end{gathered}
$$


The functions $e_{i j}(\varepsilon ; \mathbf{v})$ likewise admit in $\Omega^{m}$ a formal asymptotic expansion of the form:

$$
e_{i j}(\varepsilon ; \mathbf{v})=\frac{1}{\varepsilon} e_{i j}^{-1}(\mathbf{v})+e_{i j}^{0}(\mathbf{v})+\varepsilon e_{i j}^{1}(\mathbf{v})+\varepsilon^{2} e_{i j}^{2}(\mathbf{v})+\ldots,
$$

where

$$
\begin{gathered}
\left\{\begin{array} { l } 
{ e _ { \alpha \beta } ^ { - 1 } ( \mathbf { v } ) : = 0 , } \\
{ e _ { \alpha 3 } ^ { - 1 } ( \mathbf { v } ) : = \frac { 1 } { 2 } \partial _ { 3 } v _ { \alpha } , } \\
{ e _ { 3 3 } ^ { - 1 } ( \mathbf { v } ) : = \partial _ { 3 } v _ { 3 } , }
\end{array} \quad \left\{\begin{array}{l}
e_{\alpha \beta}^{0}(\mathbf{v}):=\frac{1}{2}\left(\partial_{\beta} v_{\alpha}+\partial_{\alpha} v_{\beta}\right)-\Gamma_{\alpha \beta}^{\sigma} v_{\sigma}-b_{\alpha \beta} v_{3}, \\
e_{\alpha 3}^{0}(\mathbf{v}):=\frac{1}{2} \partial_{\alpha} v_{3}+b_{\alpha}^{\sigma} v_{\sigma}, \\
e_{33}^{0}(\mathbf{v}):=0
\end{array}\right.\right. \\
\left\{\begin{array}{l}
e_{\alpha \beta}^{1}(\mathbf{v}):=\left.x_{3} b_{\beta}^{\sigma}\right|_{\alpha} v_{\sigma}+x_{3} b_{\alpha}^{\sigma} b_{\sigma \beta} v_{3}, \\
e_{\alpha 3}^{1}(\mathbf{v}):=x_{3} b_{\alpha}^{\tau} b_{\tau}^{\sigma} v_{\sigma}, \\
e_{33}^{1}(\mathbf{v}):=0 .
\end{array}\right.
\end{gathered}
$$

Hence, by substituting (2), (3), (7)-(11) in (6) and by identifying the terms with identical power, we can characterize the formal limit problems for $p=1$ and $p=3$.

\section{The limit problem for $p=1$}

The formulation of the limit problem when the rigidity of the shell is $\frac{1}{\varepsilon}$ is stated in the following theorem:

Theorem 1 The leading term $\mathbf{u}^{0}$ of the asymptotic expansion (7) satisfies the following variational problem:

$$
\left\{\begin{array}{l}
\text { Find } \mathbf{u}^{0} \in V_{M} \text { such that } \\
A^{-}\left(\mathbf{u}^{0}, \mathbf{v}\right)+A^{+}\left(\mathbf{u}^{0}, \mathbf{v}\right)+A_{M}^{m}\left(\mathbf{u}^{0}, \mathbf{v}\right)=L(\mathbf{v}) \text { for all } \mathbf{v} \in V_{M}
\end{array}\right.
$$

with

$$
\begin{aligned}
V_{M}:=\left\{\mathbf{v} \in L^{2}\left(\Omega ; \mathbb{R}^{3}\right) ;\right. & \mathbf{v}^{ \pm} \in H^{1}\left(\Omega^{ \pm} ; \mathbb{R}^{3}\right), v_{\alpha}^{m} \in H^{1}\left(\Omega^{m}\right), \\
& \left.L^{2}\left(\Omega^{m} ; \mathbb{R}^{3}\right) \ni \partial_{3} \mathbf{v}^{m}=\mathbf{0}, \mathbf{v}_{\mid S^{ \pm}}^{ \pm}=\mathbf{v}_{\mid S^{ \pm}}^{m}, \mathbf{v}_{\mid \Gamma_{0}}=\mathbf{0}\right\},
\end{aligned}
$$

and where

$$
\begin{gathered}
A_{M}^{m}\left(\mathbf{u}^{0}, \mathbf{v}\right):=\int_{\Omega^{m}} a^{\alpha \beta \sigma \tau} e_{\sigma \tau}^{0}\left(\mathbf{u}^{0}\right) e_{\alpha \beta}^{0}(\mathbf{v}) \sqrt{a} d x \\
a^{\alpha \beta \sigma \tau}:=\frac{2 \lambda^{m} \mu^{m}}{\lambda^{m}+2 \mu^{m}} a^{\alpha \beta} a^{\sigma \tau}+2 \mu^{m}\left(a^{\alpha \sigma} a^{\beta \tau}+a^{\alpha \tau} a^{\beta \sigma}\right),
\end{gathered}
$$

are respectively the bilinear form associated with the membrane behavior of the shell and the contravariant components of the elasticity tensor of the shell. 
Proof For convenience we split the proof in three parts numbered form $(i)$ to (iii).

(i) The variational problem corresponding to the order $\varepsilon^{-2}$ in the problem (6) is:

$$
\int_{\Omega^{m}} A_{m}^{i j k \ell}(0) e_{k \ell}^{-1} e_{i j}^{-1}(\mathbf{v}) \sqrt{a} d x=0 \text { for all } \mathbf{v} \in V
$$

The matrix $\left(a^{\alpha \beta}\right)$ being positive definite this implies that $\partial_{3} \mathbf{u}^{0}=0$ in $\Omega^{m}$. Thus the leading term $\mathbf{u}^{0}$ is independent of the transverse variable $x_{3}$ in $\Omega^{m}$ and consequently $e_{i j}^{-1}=0$.

(ii) The relations $e_{i j}^{-1}=0$ (obtained in step $(i)$ ) lead to the following variational problem associated with the order $\varepsilon^{-1}$ :

$$
\int_{\Omega^{m}} A_{m}^{i j k \ell}(0) e_{k \ell}^{0} e_{i j}^{-1}(\mathbf{v}) \sqrt{a} d x=0 \text { for all } \mathbf{v} \in V
$$

It turns out that

$$
e_{\alpha 3}^{0}=0 \text { and } e_{33}^{0}=-\frac{A^{\alpha \beta 33}(0)}{A^{3333}(0)} e_{\alpha \beta}^{0} \text { in } \Omega^{m} .
$$

(iii) The variational problem associated with the order $\varepsilon^{0}$ is:

$$
\begin{aligned}
& \int_{\Omega^{+}} A_{+}^{i j k \ell} e_{k \ell}\left(\mathbf{u}^{0}\right) e_{i j}(\mathbf{v}) \sqrt{g^{+}} d x+\int_{\Omega^{-}} A_{-}^{i j k \ell} e_{k \ell}\left(\mathbf{u}^{0}\right) e_{i j}(\mathbf{v}) \sqrt{g^{-}} d x+ \\
& +\int_{\Omega^{m}}\left\{A_{m}^{i j k \ell}(0)\left(e_{k \ell}^{0} e_{i j}^{0}(\mathbf{v})+e_{k \ell}^{1} e_{i j}^{-1}(\mathbf{v})\right)+B_{m}^{i j k \ell, 1} e_{k \ell}^{0} e_{i j}^{-1}(\mathbf{v})\right\} \sqrt{a} d x=L(\mathbf{v}),
\end{aligned}
$$

for all $\mathbf{v} \in V$. By choosing test function $\mathbf{v}$ such that $\mathbf{v}^{m}$ is independent of $x_{3}$, then $e_{i j}^{-1}(\mathbf{v})=0$ in $\Omega^{m}$ and the above variational problem reduces to:

$$
\begin{gathered}
\int_{\Omega^{+}} A_{+}^{i j k \ell} e_{k \ell}\left(\mathbf{u}^{0}\right) e_{i j}(\mathbf{v}) \sqrt{g^{+}} d x+\int_{\Omega^{-}} A_{-}^{i j k \ell} e_{k \ell}\left(\mathbf{u}^{0}\right) e_{i j}(\mathbf{v}) \sqrt{g^{-}} d x \\
+\int_{\Omega^{m}} A_{m}^{i j k \ell}(0) e_{k \ell}^{0} e_{i j}^{0}(\mathbf{v}) \sqrt{a} d x=L(\mathbf{v}) \text { for all } \mathbf{v} \in V_{M}
\end{gathered}
$$

From steps (i) and (ii), we can easily prove that

$$
\int_{\Omega^{m}} A_{m}^{i j k \ell}(0) e_{k \ell}^{0} e_{i j}^{0}(\mathbf{v}) \sqrt{a} d x=\int_{\Omega^{m}} a^{\alpha \beta \sigma \tau} e_{\sigma \tau}^{0}\left(\mathbf{u}^{0}\right) e_{\alpha \beta}^{0}(\mathbf{v}) \sqrt{a} d x
$$

Hence (13) shows that $\mathbf{u}^{0}$ is the solution of the limit problem (12).

Remark 1. One can note that the space $V_{M}$ is isomorphic to $\widehat{V}_{M}:=\{\mathbf{v} \in$ $\left.H^{1}\left(\widehat{\Omega} ; \mathbb{R}^{3}\right) ; \mathbf{v}_{\mid \omega} \in H^{1}\left(\omega ; \mathbb{R}^{2}\right) \times H^{\frac{1}{2}}(\omega), \mathbf{v}_{\mid \Gamma_{0}}=\mathbf{0}\right\}$, where $\widehat{\Omega}:=\Omega^{+} \cup \omega \cup \Omega^{-}$. 
Since $\mathbf{u}^{0}$ and $\mathbf{v}$ are independent of $x_{3}, e_{\sigma \tau}^{0}\left(\mathbf{u}^{0}\right)=\gamma_{\sigma \tau}\left(\mathbf{u}^{0}\right)$ and $e_{\alpha \beta}^{0}(\mathbf{v})=\gamma_{\alpha \beta}(\mathbf{v})$. Consequently, by integrating along the $x_{3}$-coordinate, we can write

$$
A_{M}^{m}\left(\mathbf{u}^{0}, \mathbf{v}\right)=2 \int_{\omega} a^{\alpha \beta \sigma \tau} \gamma_{\sigma \tau}\left(\mathbf{u}^{0}\right) \gamma_{\alpha \beta}(\mathbf{v}) \sqrt{a} d \widetilde{x}
$$

We obtain in the simplified model a membrane transmission condition at the interface between the two three-dimensional bodies. This condition can be interpreted as a curvilinear generalization of the Ventcel-type transmission condition obtained in [2]. Indeed, one has

$$
\left\{\begin{array} { l l } 
{ - \sigma _ { \pm } ^ { i j } \| _ { j } = f ^ { i } } & { \text { in } \Omega ^ { \pm } , } \\
{ \mathbf { u } = \mathbf { 0 } } & { \text { on } \Gamma _ { 0 } , }
\end{array} \quad \left\{\begin{array}{ll}
\llbracket \sigma^{\alpha 3} \rrbracket=\left.n^{\alpha \beta}\right|_{\beta} & \text { on } \omega \\
\llbracket \sigma^{33} \rrbracket=n^{\alpha \beta} b_{\alpha \beta} & \text { on } \omega \\
\llbracket \mathbf{u} \rrbracket=\mathbf{0} & \text { on } \omega
\end{array}\right.\right.
$$

where $\sigma_{ \pm}^{i j}:=A_{ \pm}^{i j k \ell} e_{k \ell}\left(\mathbf{u}^{ \pm}\right)$and $n^{\alpha \beta}:=a^{\alpha \beta \sigma \tau} \gamma_{\sigma \tau}\left(\mathbf{u}_{\mid \omega}\right)$ are respectively the contravariant components of the stress tensor and of the membrane stress tensor of the shell, $\llbracket \sigma^{i 3} \rrbracket:=\sigma_{+}^{i 3}-\sigma_{-}^{i 3}$ represents the stress jump at the interface $\omega$ between $\Omega^{+}$and $\Omega^{-}, \llbracket \mathbf{u} \rrbracket$ represents the displacement jump at $\omega$ between $\Omega^{+}$and $\Omega^{-}$.

\section{The limit problem for $p=3$}

We state in Theorem 2 below the formulation of the limit problem when the rigidity of the shell is $\frac{1}{\varepsilon^{3}}$.

Theorem 2 The leading term $\mathbf{u}^{0}$ of the asymptotic expansion (7) satisfies the following variational problem:

$$
\left\{\begin{array}{l}
\text { Find } \mathbf{u}^{0} \in V_{F} \text { such that } \\
A^{-}\left(\mathbf{u}^{0}, \mathbf{v}\right)+A^{+}\left(\mathbf{u}^{0}, \mathbf{v}\right)+A_{F}^{m}\left(\mathbf{u}^{0}, \mathbf{v}\right)=L(\mathbf{v}) \text { for all } \mathbf{v} \in V_{F},
\end{array}\right.
$$

where

$V_{F}:=\left\{\mathbf{v} \in H^{1}\left(\Omega ; \mathbb{R}^{3}\right) ; v_{3}^{m} \in H^{2}\left(\Omega^{m}\right), \gamma_{\alpha \beta}\left(\mathbf{v}^{m}\right)=0, \partial_{3} \mathbf{v}^{m}=\mathbf{0}, \mathbf{v}_{\mid \Gamma_{0}}=\mathbf{0}\right\}$,

and

$$
A_{F}^{m}\left(\mathbf{u}^{0}, \mathbf{v}\right):=\int_{\Omega^{m}} x_{3}^{2} a^{\alpha \beta \sigma \tau} \rho_{\sigma \tau}\left(\mathbf{u}^{0}\right) \rho_{\alpha \beta}(\mathbf{v}) \sqrt{a} d x
$$

is the bilinear form associated with the flexural behavior of the shell.

Proof The proof is divided into four steps numbered form $(i)$ to $(i v)$ and follows [5], chap. 3 . 
(i) The variational problems at order $\varepsilon^{-4}$ and $\varepsilon^{-3}$ are respectively analogous to those of steps $(i)$ and $(i i)$ of Theorem 1. Hence, one has:

$$
\begin{aligned}
& \partial_{3} \mathbf{u}^{0}=0, \\
& e_{\alpha 3}^{0}=0 \text { and } e_{33}^{0}=-\frac{A^{\alpha \beta 33}(0)}{A^{3333}(0)} e_{\alpha \beta}^{0}
\end{aligned}
$$

in $\Omega^{m}$. For $\mathbf{v} \in V$ such that $e_{i j}^{-1}(\mathbf{v})=0$, the variational problem at order $\varepsilon^{-2}$ takes the following form:

$$
\int_{\Omega^{m}} a^{\alpha \beta \sigma \tau} e_{\sigma \tau}^{0} e_{\alpha \beta}^{0}(\mathbf{v}) \sqrt{a} d x=0 .
$$

Thus $e_{\alpha \beta}^{0}=0$.

(ii) Since $e_{\alpha \beta}^{0}=\gamma_{\alpha \beta}\left(\mathbf{u}^{0}\right)=0$, from $\left(15_{2}\right)$ it turns out that:

$$
e_{33}^{0}=\partial_{3} u_{3}^{1}=0 \text { and } e_{\alpha 3}^{0}=\frac{1}{2}\left(\partial_{3} u_{\alpha}^{1}+\partial_{\alpha} u_{3}^{0}\right)+b_{\alpha}^{\sigma} u_{\sigma}^{0}=0 .
$$

Hence

$$
u_{\alpha}^{1}(x)=\bar{u}_{\alpha}^{1}(\widetilde{x})-x_{3}\left(\partial_{\alpha} u_{3}^{0}+2 b_{\alpha}^{\tau} u_{\tau}^{0}\right)(\widetilde{x}) \text { and } u_{3}^{1}(x)=\bar{u}_{3}^{1}(\widetilde{x}) \text { in } \Omega^{m} .
$$

From the assumption on the asymptotic expansion (7) it follows that $u_{3}^{0} \in$ $H^{2}\left(\Omega^{m}\right)$. Since $e_{i j}^{0}=0$, the variational problem at order $\varepsilon^{-2}$ reduces to

$$
\int_{\Omega^{m}} A_{m}^{i j k \ell}(0) e_{k \ell}^{1} e_{i j}^{-1}(\mathbf{v}) \sqrt{a} d x=0 \text { for all } \mathbf{v} \in V .
$$

By similar computations as in Theorem 1, we deduce that

$$
e_{\alpha 3}^{1}=0 \text { and } e_{33}^{1}=-\frac{A^{\alpha \beta 33}(0)}{A^{3333}(0)} e_{\alpha \beta}^{1} .
$$

Using (9) one can express $e_{\alpha \beta}^{1}$ in terms of $\overline{\mathbf{u}}^{1}$ and $\mathbf{u}^{0}$. An easy computation gives $e_{\alpha \beta}^{1}=e_{\alpha \beta}^{0}\left(\overline{\mathbf{u}}^{1}\right)-x_{3} \rho_{\alpha \beta}\left(\mathbf{u}^{0}\right)$.

(iii) The variational problem at order $\varepsilon^{-1}$ takes the form (we recall that $\left.e_{i j}^{-1}=e_{i j}^{0}=0\right)$ :

$$
\int_{\Omega^{m}}\left\{A_{m}^{i j k \ell}(0)\left(e_{k \ell}^{1} e_{i j}^{0}(\mathbf{w})+e_{k \ell}^{2} e_{i j}^{-1}(\mathbf{w})\right) \sqrt{a}+B_{m}^{i j k \ell, 1} e_{k \ell}^{1} e_{i j}^{-1}(\mathbf{w})\right\} d x=0
$$

for all $\mathbf{w} \in V$.

Let choose again $\mathbf{w}$ such that $e_{i j}^{-1}(\mathbf{w})=0$. Then from (16),

$$
\begin{aligned}
& \int_{\Omega^{m}} A_{m}^{i j k \ell}(0) e_{k \ell}^{1} e_{i j}^{0}(\mathbf{w}) \sqrt{a} d x=\int_{\Omega^{m}} a^{\alpha \beta \sigma \tau} e_{\sigma \tau}^{1} e_{\alpha \beta}^{0}(\mathbf{w}) \sqrt{a} d x= \\
& =\int_{\Omega^{m}} a^{\alpha \beta \sigma \tau} e_{\sigma \tau}^{0}\left(\overline{\mathbf{u}}^{1}\right) e_{\alpha \beta}^{0}(\mathbf{w}) \sqrt{a} d x=0 .
\end{aligned}
$$


Therefore $e_{\sigma \tau}^{0}\left(\overline{\mathbf{u}}^{1}\right)=0$ and so $e_{\sigma \tau}^{1}=-x_{3} \rho_{\sigma \tau}\left(\mathbf{u}^{0}\right)$.

(iv) The problem at order $\varepsilon^{0}$ takes the following form:

$$
\begin{aligned}
& \int_{\Omega^{+}} A_{+}^{i j k \ell} e_{k \ell}\left(\mathbf{u}^{0}\right) e_{i j}(\mathbf{v}) \sqrt{g^{+}} d x+\int_{\Omega^{-}} A_{-}^{i j k \ell} e_{k \ell}\left(\mathbf{u}^{0}\right) e_{i j}(\mathbf{v}) \sqrt{g^{-}} d x+ \\
& +\int_{\Omega^{m}} A_{m}^{i j k \ell}(0)\left(e_{k \ell}^{1} e_{i j}^{1}(\mathbf{v})+e_{k \ell}^{2} e_{i j}^{0}(\mathbf{v})+e_{k \ell}^{3} e_{i j}^{-1}(\mathbf{v})\right) \sqrt{a} d x+ \\
& +\int_{\Omega^{m}} B_{m}^{i j k \ell, 1}\left(e_{k \ell}^{1} e_{i j}^{0}(\mathbf{v})+e_{k \ell}^{2} e_{i j}^{-1}(\mathbf{v})\right) d x \\
& +\int_{\Omega^{m}}^{i j k \ell, 2} B_{m}^{1} e_{i j}^{-1}(\mathbf{v}) d x=L(\mathbf{v}) \text { for all } \mathbf{v} \in V .
\end{aligned}
$$

By subtracting equation (17) one obtains:

$$
\begin{aligned}
& \int_{\Omega^{+}} A_{+}^{i j k \ell} e_{k \ell}\left(\mathbf{u}^{0}\right) e_{i j}(\mathbf{v}) \sqrt{g^{+}} d x+\int_{\Omega^{-}} A_{-}^{i j k \ell} e_{k \ell}\left(\mathbf{u}^{0}\right) e_{i j}(\mathbf{v}) \sqrt{g^{-}} d x+ \\
& +\int_{\Omega^{m}} A_{m}^{i j k \ell}(0)\left\{\left(e_{k \ell}^{1}\left(e_{i j}^{1}(\mathbf{v})-e_{i j}^{0}(\mathbf{w})\right)+e_{k \ell}^{2}\left(e_{i j}^{0}(\mathbf{v})-e_{i j}^{-1}(\mathbf{w})\right)\right.\right. \\
& \left.\left.+e_{k \ell}^{3} e_{i j}^{-1}(\mathbf{v})\right) \sqrt{a}\right\} d x+ \\
& +\int_{\Omega^{m}} B_{m}^{i j k \ell, 1}\left\{\left(e_{k \ell}^{1}\left(e_{i j}^{0}(\mathbf{v})-e_{i j}^{-1}(\mathbf{w})\right)+e_{k \ell}^{2} e_{i j}^{-1}(\mathbf{v})\right)\right\} d x \\
& +\int_{\Omega^{m}} B_{m}^{i j k \ell, 2} e_{k \ell}^{1} e_{i j}^{-1}(\mathbf{v}) d x=L(\mathbf{v}) \text { for all } \mathbf{v}, \mathbf{w} \in V .
\end{aligned}
$$

Given an arbitrary test function $\mathbf{v} \in V_{F}$, let choose $\mathbf{w} \in V$ such that

$$
w_{\alpha}=x_{3}\left(2 b_{\alpha}^{\tau} v_{\tau}+\partial_{\alpha} v_{3}\right) \text { and } w_{3}=0 \text { in } \Omega^{m} .
$$

Since $e_{i j}^{-1}(\mathbf{v})=0$ and $e_{\alpha \beta}^{1}(\mathbf{v})-e_{\alpha \beta}^{0}(\mathbf{w})=-x_{3} \rho_{\alpha \beta}(\mathbf{v}), e_{\alpha \beta}^{0}(\mathbf{v})-e_{\alpha \beta}^{-1}(\mathbf{w})=0$ in $\Omega^{m}$, it follows the desired result.

Remark 1. Note that the space $V_{F}$ is isomorphic to $\widehat{V}_{F}:=\left\{\mathbf{v} \in H^{1}\left(\widehat{\Omega} ; \mathbb{R}^{3}\right) ; \mathbf{v}_{\mid \omega} \in\right.$ $H^{1}\left(\omega ; \mathbb{R}^{2}\right) \times H^{2}(\omega), \mathbf{v}_{\mid \Gamma_{0}}=\mathbf{0}, \gamma_{\alpha \beta}\left(\mathbf{v}_{\mid \omega}\right)=0$ in $\left.\omega\right\}$. Since $\mathbf{u}^{0}$ and $\mathbf{v}$ are independent of $x_{3}, \rho_{\sigma \tau}\left(\mathbf{u}^{0}\right)$ and $\rho_{\sigma \tau}(\mathbf{v})$ are also independent of $x_{3}$. Consequently, by integrating along the $x_{3}$-coordinate, we get

$$
A_{F}^{m}\left(\mathbf{u}^{0}, \mathbf{v}\right)=\frac{2}{3} \int_{\omega} a^{\alpha \beta \sigma \tau} \rho_{\sigma \tau}\left(\mathbf{u}^{0}\right) \rho_{\alpha \beta}(\mathbf{v}) \sqrt{a} d \widetilde{x} .
$$

The simplified model is characterized by a flexural transmission condition at the interface between the two three-dimensional bodies as follows:

\section{Elasticity problems in $\Omega^{ \pm} \quad$ Transmission conditions on $\omega$}

$$
\left\{\begin{array} { l l } 
{ - \sigma _ { \pm } ^ { i j } \| _ { j } = f ^ { i } } & { \text { in } \Omega ^ { \pm } , } \\
{ \mathbf { u } = \mathbf { 0 } } & { \text { on } \Gamma _ { 0 } , }
\end{array} \quad \left\{\begin{array}{ll}
\llbracket \sigma^{\alpha 3} \rrbracket=\left.\left(b_{\sigma}^{\alpha} m^{\sigma \beta}\right)\right|_{\beta}+b_{\sigma}^{\alpha}\left(\left.m^{\sigma \beta}\right|_{\beta}\right) & \text { on } \omega \\
\llbracket \sigma^{33} \rrbracket=b_{\alpha}^{\sigma} b_{\sigma \beta} m^{\alpha \beta}-\left.m^{\alpha \beta}\right|_{\alpha \beta} & \text { on } \omega \\
\llbracket \mathbf{u} \rrbracket=\mathbf{0} & \text { on } \omega
\end{array}\right.\right.
$$

where $m^{\alpha \beta}:=\frac{1}{3} a^{\alpha \beta \sigma \tau} \rho_{\sigma \tau}\left(\mathbf{v}_{\mid \omega}\right)$ are the contravariant components of the moment tensor of the shell. 


\section{Two Korn's type results}

In the whole paper, we denote by $\|\cdot\|_{s, \Omega}$ the norm in the Sobolev space $H^{s}\left(\Omega, \mathbb{R}^{d}\right)$ for every $d \geq 1$ and $\|\cdot\|_{0, \Omega}$ will stand for the norm in $L^{2}\left(\Omega, \mathbb{R}^{d}\right)$. Obviously, the same holds in $\Omega^{ \pm}, \Omega^{m}, \omega$.

Let us recall that $V=\left\{\mathbf{v} \in H^{1}\left(\Omega ; \mathbb{R}^{3}\right) ; \quad \mathbf{v}_{\mid \Gamma_{0}}=\mathbf{0}\right\}$. In order to study convergence of the solutions of problems (6) for $p \in\{1,3\}$, we establish the two following Korn type inequalities in curvilinear coordinates.

Proposition 1 There exists a constant $C>0$ such that for $\varepsilon$ small enough and for all $\mathbf{v} \in V$ :

$$
\|\mathbf{v}\|_{1, \Omega} \leq C\left\{\left\|e_{i j}(\mathbf{v})\right\|_{0, \Omega^{+}}^{2}+\left\|e_{i j}(\mathbf{v})\right\|_{0, \Omega^{-}}^{2}+\frac{1}{\varepsilon^{2}}\left\|e_{i j}(\varepsilon ; \mathbf{v})\right\|_{0, \Omega^{m}}^{2}\right\}^{1 / 2} .
$$

Proof Assume that the announced inequality is false. Then there exist $\varepsilon_{k} \rightarrow 0$ and $\left(\mathbf{v}^{k}\right)_{k=1}^{\infty} \in V$ such that

$$
\begin{array}{ll}
\left\|\mathbf{v}^{k}\right\|_{1, \Omega}=1 & \text { for all } k, \\
e_{i j}\left(\mathbf{v}^{k}\right) \rightarrow 0 & \text { in } L^{2}\left(\Omega^{ \pm}\right), \\
\frac{1}{\varepsilon_{k}} e_{i j}\left(\varepsilon_{k} ; \mathbf{v}^{k}\right) \rightarrow 0 & \text { in } L^{2}\left(\Omega^{m}\right) .
\end{array}
$$

Therefore there exist $\mathbf{v} \in V$ and a subsequence (not relabeled) such that

$$
\mathbf{v}^{k} \rightarrow \mathbf{v} \text { in } H^{1}\left(\Omega ; \mathbb{R}^{3}\right),
$$

and thus $\mathbf{v}^{k} \rightarrow \mathbf{v}$ in $L^{2}\left(\Omega ; \mathbb{R}^{3}\right)$. Moreover, from the convergence $e_{i j}\left(\mathbf{v}^{k}\right) \rightarrow 0$ in $L^{2}\left(\Omega^{+}\right)$we deduce that $\mathbf{v}^{k} \rightarrow \mathbf{v}=\mathbf{0}$ in $H^{1}\left(\Omega^{+} ; \mathbb{R}^{3}\right)$. Besides, one has

$$
\begin{array}{ll}
\frac{1}{\varepsilon_{k}^{2}} \partial_{3} v_{3}^{k}=\frac{1}{\varepsilon_{k}} e_{33}\left(\varepsilon_{k} ; \mathbf{v}^{k}\right) \rightarrow 0 & \text { in } L^{2}\left(\Omega^{m}\right), \\
\partial_{3} v_{\alpha}^{k}=\varepsilon_{k}\left(2 e_{\alpha 3}\left(\varepsilon_{k} ; \mathbf{v}^{k}\right)-\partial_{\alpha} v_{3}^{k}+2 \Gamma_{\alpha 3}^{\sigma}\left(\varepsilon_{k}\right) v_{\sigma}^{k}\right) \rightarrow 0 & \text { in } L^{2}\left(\Omega^{m}\right) .
\end{array}
$$

Thus, $\partial_{3} \mathbf{v}=\mathbf{0}$ in $\Omega^{m}$. Then, from the continuity of the trace on $S^{+}$, we deduce that $\mathbf{v}=\mathbf{0}$ in $\Omega^{m}$. Finally the convergence $e_{i j}\left(\mathbf{v}^{k}\right) \rightarrow 0$ in $L^{2}\left(\Omega^{-}\right)$ and the continuity of the trace on $S^{-}$imply that $\mathbf{v}=\mathbf{0}$ in $\Omega^{-}$. Hence we have that $\mathbf{v}^{k} \rightarrow \mathbf{0}=\mathbf{v}$ in $H^{1}\left(\Omega^{ \pm} ; \mathbb{R}^{3}\right)$. In order to conclude one has to prove that $\mathbf{v}^{k} \rightarrow \mathbf{0}=\mathbf{v}$ in $H^{1}\left(\Omega^{m} ; \mathbb{R}^{3}\right)$. We remark at first that $e_{i j}\left(\varepsilon_{k} ; \mathbf{v}^{k}\right) \rightarrow 0$ in $L^{2}\left(\Omega^{m}\right)$ implies that $\partial_{1} v_{1}^{k}, \partial_{2} v_{2}^{k}$ and $\partial_{1} v_{2}^{k}+\partial_{2} v_{1}^{k}$ tend to zero strongly in $L^{2}\left(\Omega^{m}\right)$. As in the classical proof of Korn's inequality, one deduces that $\partial_{1} v_{2}^{k}$ and $\partial_{2} v_{1}^{k}$ tend to zero strongly in $L^{2}\left(\Omega^{m}\right)$. To prove that $\partial_{\alpha} v_{3}^{k}$ tends to zero strongly in $L^{2}\left(\Omega^{m}\right)$ it is enough to prove that $\partial_{\alpha \beta} v_{3}^{k}$ tends to zero in $H^{-1}\left(\Omega^{m}\right)$. Let us remark that

$$
\begin{aligned}
\partial_{\alpha \beta} v_{3}^{k}= & \partial_{\beta} e_{\alpha 3}\left(\varepsilon_{k} ; \mathbf{v}^{k}\right)+\partial_{\alpha} e_{\beta 3}\left(\varepsilon_{k} ; \mathbf{v}^{k}\right)-\frac{1}{\varepsilon_{k}} \partial_{3} e_{\alpha \beta}\left(\varepsilon_{k} ; \mathbf{v}^{k}\right)-\frac{1}{\varepsilon_{k}} \partial_{3}\left(\Gamma_{\alpha \beta}^{p}\left(\varepsilon_{k}\right) v_{p}^{k}\right) \\
& +\Gamma_{\alpha 3}^{\sigma}\left(\varepsilon_{k}\right) \partial_{\beta} v_{\sigma}^{k}+\Gamma_{\beta 3}^{\sigma}\left(\varepsilon_{k}\right) \partial_{\alpha} v_{\sigma}^{k}+\left(\partial_{\beta} \Gamma_{\alpha 3}^{\sigma}\left(\varepsilon_{k}\right)+\partial_{\alpha} \Gamma_{\beta 3}^{\sigma}\left(\varepsilon_{k}\right)\right) v_{\alpha}^{k} .
\end{aligned}
$$

From the previous considerations, one immediately deduces that all the terms in the right-hand side go to zero in $H^{-1}\left(\Omega^{m} ; \mathbb{R}^{3}\right)$ except $\frac{1}{\varepsilon_{k}} \partial_{3}\left(\Gamma_{\alpha \beta}^{p}\left(\varepsilon_{k}\right) v_{p}^{k}\right)$. By 
using (3) and the previous results, one sees that it is enough to prove that $\frac{1}{\varepsilon_{k}} \partial_{3} v^{k}$ tends to zero in $H^{-1}\left(\Omega^{m} ; \mathbb{R}^{3}\right)$. This in turn follows from the definition of $e_{\alpha 3}\left(\varepsilon_{k} ; \mathbf{v}^{k}\right)$ and the convergence of $v_{3}^{k}$ to zero in $L^{2}\left(\Omega^{m}\right)$. Hence the sequence $\left(\mathbf{v}^{k}\right)_{k=1}^{\infty}$ converges strongly to $\mathbf{0}$ in $H^{1}\left(\Omega ; \mathbb{R}^{3}\right)$ which is contradictory with the assumption $\left\|\mathbf{v}^{k}\right\|_{1, \Omega}=1$ for all $k$.

Let us define the space $\mathcal{V}$ :

$$
\begin{aligned}
\mathcal{V}:=\left\{\mathbf{v} \in L^{2}\left(\Omega ; \mathbb{R}^{3}\right) ; \mathbf{v}^{ \pm} \in H^{1}\left(\Omega^{ \pm} ; \mathbb{R}^{3}\right), v_{\alpha}^{m} \in H^{1}\left(\Omega^{m}\right),\right. \\
\left.\partial_{3} \mathbf{v}^{m} \in L^{2}\left(\Omega^{m} ; \mathbb{R}^{3}\right), \mathbf{v}_{\mid S^{ \pm}}^{ \pm}=\mathbf{v}_{\mid S^{ \pm}}^{m}, \mathbf{v}_{\mid \Gamma_{0}}=\mathbf{0}\right\},
\end{aligned}
$$

equipped with the norm $\|\cdot\|_{\mathcal{V}}$ defined by

$$
\begin{aligned}
\|\mathbf{v}\|_{\mathcal{V}}:=\left\{\left\|e_{i j}(\mathbf{v})\right\|_{0, \Omega^{+}}^{2}\right. & +\left\|e_{i j}(\mathbf{v})\right\|_{0, \Omega^{-}}^{2}+\|\mathbf{v}\|_{0, \Omega}^{2}+ \\
& \left.+\left\|\partial_{\alpha} v_{\beta}\right\|_{0, \Omega^{m}}^{2}\left\|+\mid \partial_{3} \mathbf{v}\right\|_{0, \Omega^{m}}^{2}\right\}^{1 / 2} .
\end{aligned}
$$

We can easily prove that $\mathcal{V}$ is complete and that $V_{M} \subset \mathcal{V}$ is a closed subspace; therefore the uniqueness of the solution for problem (12) is guaranteed. Let us also remark that $V \subset \mathcal{V}$.

Proposition 2 There exists a constant $C>0$ such that for $\varepsilon$ small enough,

$\|\mathbf{v}\|_{\mathcal{V}} \leq C\left\{\left\|e_{i j}(\mathbf{v})\right\|_{0, \Omega^{+}}^{2}+\left\|e_{i j}(\mathbf{v})\right\|_{0, \Omega^{-}}^{2}+\left\|e_{i j}(\varepsilon ; \mathbf{v})\right\|_{0, \Omega^{m}}^{2}\right\}^{1 / 2}$ for all $\mathbf{v} \in \mathcal{V}$.

Proof Assume that the announced inequality is false. Then there exists $\varepsilon_{k} \rightarrow 0$ and $\left(\mathbf{v}^{k}\right)_{k=1}^{\infty} \in \mathcal{V}$ such that

$$
\begin{aligned}
& \left\|\mathbf{v}^{k}\right\|_{\mathcal{V}}=1 \quad \text { for all } k, \\
& e_{i j}\left(\mathbf{v}^{k}\right) \rightarrow 0 \quad \text { in } L^{2}\left(\Omega^{ \pm}\right), \\
& e_{i j}\left(\varepsilon_{k} ; \mathbf{v}^{k}\right) \rightarrow 0 \text { in } L^{2}\left(\Omega^{m}\right) .
\end{aligned}
$$

Up to extraction of a subsequence (not relabeled), there exists $\mathbf{v} \in \mathcal{V}$ such that

$$
\begin{array}{ll}
\mathbf{v}^{k} \rightarrow \mathbf{v} & \text { in } H^{1}\left(\Omega^{ \pm} ; \mathbb{R}^{3}\right), \\
v_{3}^{k} \rightarrow v_{3} & \text { in } L^{2}\left(\Omega^{m}\right), \\
v_{\alpha}^{k} \rightarrow v_{\alpha} & \text { in } H^{1}\left(\Omega^{m}\right), \\
\partial_{3} \mathbf{v}^{k} \rightarrow \partial_{3} \mathbf{v} & \text { in } L^{2}\left(\Omega^{m} ; \mathbb{R}^{3}\right) .
\end{array}
$$

The convergence $e_{i j}\left(\mathbf{v}^{k}\right) \rightarrow 0$ in $L^{2}\left(\Omega^{+}\right)$implies that $\mathbf{v}=\mathbf{0}$ in $\Omega^{+}$and $\mathbf{v}^{k} \rightarrow$ $\mathbf{v}=\mathbf{0}$ in $H^{1}\left(\Omega^{+} ; \mathbb{R}^{3}\right)$. Besides, one has

$$
\begin{aligned}
& \frac{1}{\varepsilon_{k}} \partial_{3} v_{3}^{k}=e_{33}\left(\varepsilon_{k} ; \mathbf{v}^{k}\right) \rightarrow 0 \text { in } L^{2}\left(\Omega^{m}\right), \\
& \partial_{3} v_{\alpha}^{k}+\varepsilon_{k} \partial_{\alpha} v_{3}^{k}=\varepsilon_{k}\left(2 e_{\alpha 3}\left(\varepsilon_{k} ; \mathbf{v}^{k}\right)+2 \Gamma_{\alpha 3}^{\sigma}\left(\varepsilon_{k}\right) v_{\sigma}^{k}\right) \rightarrow 0 \text { in } L^{2}\left(\Omega^{m}\right) .
\end{aligned}
$$

Hence for all $\varphi \in \mathcal{D}\left(\Omega^{m}\right)$, one has:

$$
\begin{aligned}
\int_{\Omega^{m}} \partial_{3} v_{\alpha} \varphi d x & =-\lim _{k \rightarrow \infty} \int_{\Omega^{m}}\left(v_{\alpha}^{k} \partial_{3} \varphi+\varepsilon_{k} v_{3}^{k} \partial_{\alpha} \varphi\right) d x \\
& =\lim _{k \rightarrow \infty} \int_{\Omega^{m}}\left(\partial_{3} v_{\alpha}^{k}+\varepsilon_{k} \partial_{\alpha} v_{3}^{k}\right) \varphi d x=0 .
\end{aligned}
$$


Thus, $\partial_{3} \mathbf{v}=\mathbf{0}$ in $\Omega^{m}$, and by the continuity of the trace on $S^{+}$, we deduce that $\mathbf{v}=\mathbf{0}$ in $\Omega^{m}$. Finally the convergence $e_{i j}\left(\mathbf{v}^{k}\right) \rightarrow 0$ in $L^{2}\left(\Omega^{-}\right)$and the continuity of the trace on $S^{-}$imply that $\mathbf{v}=\mathbf{0}$ in $\Omega^{-}$. Hence we have that $\mathbf{v}^{k} \rightarrow \mathbf{0}=\mathbf{v}$ in $H^{1}\left(\Omega^{ \pm} ; \mathbb{R}^{3}\right)$. Moreover, since

$$
\int_{\Omega^{m}}\left|v_{3}^{k}\right|^{2} d x \leq C\left(\int_{S^{+}}\left|v_{3}^{k}(\widetilde{x}, 1)\right|^{2} d \widetilde{x}+\int_{\Omega^{m}}\left|\partial_{3} v_{3}^{k}\right|^{2} d x\right),
$$

it follows that $v_{3}^{k} \rightarrow 0$ strongly in $L^{2}\left(\Omega^{m}\right)$. Let us now prove that $\partial_{3} v_{\alpha}^{k} \rightarrow 0$ in $L^{2}\left(\Omega^{m}\right)$. From (19), it is enough to establish that $\varepsilon_{k} \partial_{\alpha} v_{3}^{k}$ tends to zero in $L^{2}\left(\Omega^{m}\right)$. This can be deduced from (18) and the previous results. In order to conclude one has to prove that $v_{\alpha}^{k} \rightarrow 0$ in $H^{1}\left(\Omega^{m}\right)$. For this we apply the classical Korn inequality to $\left(\mathbf{z}^{k}\right)_{k=1}^{\infty}$ where $\mathbf{z}^{k}=\left(z_{i}^{k}\right):=\left(v_{1}^{k}, v_{2}^{k}, 0\right)$. We set $\widehat{e}_{i j}(\mathbf{z}):=\frac{1}{2}\left(\partial_{i} z_{j}+\partial_{j} z_{i}\right)$. Since $\widehat{e}_{\alpha \beta}\left(\mathbf{z}^{k}\right)=e_{\alpha \beta}\left(\varepsilon_{k} ; \mathbf{v}^{k}\right)+\Gamma_{\alpha \beta}^{p}\left(\varepsilon_{k}\right) v_{p}^{k}$ and $\widehat{e}_{\alpha 3}\left(\mathbf{z}^{k}\right)=\frac{1}{2} \partial_{3} v_{\alpha}^{k}$, it follows that $\widehat{e}_{i j}\left(\mathbf{z}^{k}\right) \rightarrow 0$ in $L^{2}\left(\Omega^{m}\right)$ and so $\mathbf{z}^{k} \rightarrow \mathbf{0}$ in $H^{1}\left(\Omega^{m}\right)$. Hence the sequence $\left(\mathbf{v}^{k}\right)_{k=1}^{\infty}$ converges strongly to $\mathbf{0}$ in $\mathcal{V}$ which is contradictory with the hypothesis $\left\|\mathbf{v}^{k}\right\|_{\mathcal{V}}=1$ for all $k$.

\section{Convergence results}

8.1 Strong convergence for $p=1$

For every function $\mathbf{v}$ defined almost everywhere over $\left.\Omega^{m}=\omega \times\right]-1,1$, we define the average

$$
\overline{\mathbf{v}}(\widetilde{x}):=\frac{1}{2} \int_{-1}^{1} \mathbf{v}\left(\widetilde{x}, x_{3}\right) d x_{3} \text { for all } \widetilde{x} \in \omega .
$$

We recall for later use that the weak convergence in $L^{2}\left(\Omega^{m} ; \mathbb{R}^{3}\right)$ implies the weak convergence of the average in $L^{2}\left(\omega ; \mathbb{R}^{3}\right)$

Let $\mathbf{u}(\varepsilon) \in V \subset \mathcal{V}$ be the solution of (6) for $p=1$. Thanks to the assumptions on the loading, the coercivity of the bilinear forms $A^{ \pm}$and $A^{m}$, the classical Korn inequality and Proposition 2, one obtains the following a priori estimates:

$$
\begin{aligned}
& \|\mathbf{u}(\varepsilon)\|_{\mathcal{V}} \leq C, \\
& \left\|e_{i j}(\varepsilon ; \mathbf{u}(\varepsilon))\right\|_{0, \Omega^{m}} \leq C .
\end{aligned}
$$

Theorem 3 The sequence $(\mathbf{u}(\varepsilon))_{\varepsilon>0}$ converges strongly in $\mathcal{V}$ to $\mathbf{u}^{0} \in V_{M}$, the unique solution of problem (12).

Proof For convenience, the proof is divided into seven parts, numbered from (i) to $($ vii).

(i) From (20) we deduce that there exist a subsequence (not relabeled), $\mathbf{u} \in \mathcal{V}$ and $e_{i j} \in L^{2}\left(\Omega^{m}\right)$ such that

$$
\begin{array}{ll}
\mathbf{u}(\varepsilon) \rightarrow \mathbf{u} & \text { in } \mathcal{V}, \\
e_{i j}(\varepsilon ; \mathbf{u}(\varepsilon)) \rightarrow e_{i j} & \text { in } L^{2}\left(\Omega^{m}\right) .
\end{array}
$$


Let us explicitly remark that $\mathbf{u}(\varepsilon) \rightarrow \mathbf{u}$ in $\mathcal{V}$ means that:

$$
\begin{array}{ll}
\mathbf{u}(\varepsilon) \rightarrow \mathbf{u} & \text { in } H^{1}\left(\Omega^{ \pm} ; \mathbb{R}^{3}\right), \\
u_{3}(\varepsilon) \rightarrow u_{3} \quad \text { in } L^{2}\left(\Omega^{m}\right), & \text { in } H^{1}\left(\Omega^{m}\right), \\
u_{\alpha}(\varepsilon) \rightarrow u_{\alpha} \quad & \partial_{3} \mathbf{u}(\varepsilon) \rightarrow \partial_{3} \mathbf{u} \text { in } L^{2}\left(\Omega^{m} ; \mathbb{R}^{3}\right) .
\end{array}
$$

(ii) We prove that $\partial_{3} \mathbf{u}=\mathbf{0}$ in $\Omega^{m}$. From (20) and (21), one has

$$
\begin{array}{ll}
\partial_{3} u_{3}(\varepsilon)=\varepsilon e_{33}(\varepsilon ; \mathbf{u}(\varepsilon)) \rightarrow 0=\partial_{3} u_{3} & \text { in } L^{2}\left(\Omega^{m}\right), \\
\partial_{3} u_{\alpha}(\varepsilon)+\varepsilon \partial_{\alpha} u_{3}(\varepsilon)=2 \varepsilon\left\{e_{\alpha 3}(\varepsilon ; \mathbf{u}(\varepsilon))+\Gamma_{\alpha 3}^{\sigma}(\varepsilon) u_{\sigma}(\varepsilon)\right\} \rightarrow 0 & \text { in } L^{2}\left(\Omega^{m}\right) .
\end{array}
$$

Thus, with the same arguments as in the proof of Proposition 2, we obtain $\partial_{3} u_{\alpha}=0$ in $L^{2}\left(\Omega^{m}\right)$ and $\mathbf{u} \in V_{M}$.

(iii) The limits $e_{\alpha \beta}$ satisfy the relation $\overline{e_{\alpha \beta}}=\gamma_{\alpha \beta}(\mathbf{u})$. Indeed using the definition of the average of $e_{\alpha \beta}(\varepsilon ; \mathbf{u}(\varepsilon))$, of $\gamma_{\alpha \beta}(\overline{\mathbf{u}(\varepsilon)})$ and of $\Gamma_{\alpha \beta}^{\sigma}(\varepsilon)$, we deduce that

$$
\left\|\overline{e_{\alpha \beta}(\varepsilon ; \mathbf{u}(\varepsilon))}-\gamma_{\alpha \beta}(\overline{\mathbf{u}(\varepsilon)})\right\|_{0, \omega} \leq C \varepsilon\|\mathbf{u}(\varepsilon)\|_{0, \Omega^{m}}
$$

which tends to zero as $\varepsilon \rightarrow 0$. On the other hand, by definition of $\mathcal{V}, \gamma_{\alpha \beta}(\mathbf{u}(\varepsilon)) \rightarrow$ $\gamma_{\alpha \beta}(\mathbf{u})$ in $L^{2}\left(\Omega^{m}\right)$ which implies that $\gamma_{\alpha \beta}(\overline{\mathbf{u}(\varepsilon)}) \rightarrow \gamma_{\alpha \beta}(\overline{\mathbf{u}})=\gamma_{\alpha \beta}(\mathbf{u})$ in $L^{2}(\omega)$ and thus $\overline{e_{\alpha \beta}}=\gamma_{\alpha \beta}(\mathbf{u})$ in $L^{2}(\omega)$.

(iv) The limits $e_{\alpha \beta}$ satisfy $\partial_{3} e_{\alpha \beta}=0$. For this let us now remark that

$$
\partial_{3} e_{\alpha \beta}(\varepsilon ; \mathbf{u}(\varepsilon))=\frac{1}{2}\left(\partial_{\beta} \partial_{3} u_{\alpha}(\varepsilon)+\partial_{\alpha} \partial_{3} u_{\beta}(\varepsilon)\right)-\partial_{3}\left(\Gamma_{\alpha \beta}^{p}(\varepsilon) u_{p}(\varepsilon)\right) .
$$

Thanks to (ii), (21) and (3), the right-hand side converges to zero weakly in $H^{-1}\left(\Omega^{m}\right)$ as $\varepsilon \rightarrow 0$. The continuity of the operator $\partial_{3}: L^{2}\left(\Omega^{m}\right) \rightarrow H^{-1}(\Omega)$ implies that $\partial_{3} e_{\alpha \beta}=0$ and so

$$
e_{\alpha \beta}=\gamma_{\alpha \beta}(\mathbf{u}) \text { in } L^{2}\left(\Omega^{m}\right)
$$

From (8) and (4) we finally obtain $e_{\alpha \beta}=e_{\alpha \beta}^{0}(\mathbf{u})$.

$(v)$ By multiplying problem (6) by $\varepsilon$ and by letting $\varepsilon \rightarrow 0$, we get

$$
e_{\alpha 3}=0 \text { and } e_{33}=-\frac{A^{\alpha \beta 33}(0)}{A^{3333}(0)} e_{\alpha \beta} .
$$

By choosing in (6) test functions $\mathbf{v}$ independent of $x_{3}$ in $\Omega^{m}$ and by applying the limit as $\varepsilon \rightarrow 0$, we obtain:

$A^{+}(\mathbf{u}, \mathbf{v})+A^{-}(\mathbf{u}, \mathbf{v})+\int_{\Omega^{m}}\left(A^{\alpha \beta \sigma \tau}(0) e_{\alpha \beta} e_{\sigma \tau}^{0}(\mathbf{v})+A^{\sigma \tau 33}(0) e_{33} e_{\sigma \tau}^{0}(\mathbf{v})\right) d x=L(\mathbf{v})$.

From (23) and (24), we infer that

$$
A^{+}(\mathbf{u}, \mathbf{v})+A^{-}(\mathbf{u}, \mathbf{v})+\int_{\Omega^{m}} a^{\alpha \beta \sigma \tau} e_{\alpha \beta}^{0}(\mathbf{u}) e_{\sigma \tau}^{0}(\mathbf{v}) \sqrt{a} d x=L(\mathbf{v}) .
$$


Hence, by virtue of the uniqueness of the solution, we deduce that $\mathbf{u}=\mathbf{u}^{0}$.

(vi) Let us prove the strong convergence of $e_{i j}(\varepsilon ; \mathbf{u}(\varepsilon))$ to $e_{i j}$ in $L^{2}\left(\Omega^{m}\right)$. For this we remark that

$$
\begin{aligned}
& \int_{\Omega^{+}} A_{+}^{i j k \ell}\left(e_{k \ell}(\mathbf{u}(\varepsilon))-e_{k \ell}\right)\left(e_{i j}(\mathbf{u}(\varepsilon))-e_{i j}\right) \sqrt{g^{+}} d x+ \\
& +\int_{\Omega^{-}} A_{-}^{i j k \ell}\left(e_{k \ell}(\mathbf{u}(\varepsilon))-e_{k \ell}\right)\left(e_{i j}(\mathbf{u}(\varepsilon))-e_{i j}\right) \sqrt{g^{-}} d x+ \\
& +\int_{\Omega^{m}} A_{m}^{i j k \ell}\left(e_{k \ell}(\varepsilon ; \mathbf{u}(\varepsilon))-e_{k \ell}\right)\left(e_{i j}(\varepsilon ; \mathbf{u}(\varepsilon))-e_{i j}\right) \sqrt{g^{m}} d x
\end{aligned}
$$

tends to zero. From the coercivity, we obtain the claimed strong convergence. Moreover, from the classical Korn inequality, we deduce the strong convergence of $\mathbf{u}^{ \pm}(\varepsilon)$ to $\mathbf{u}^{0, \pm}$ in $H^{1}\left(\Omega^{ \pm} ; \mathbb{R}^{3}\right)$.

(vii) In order to conclude the proof of the strong convergence of $\mathbf{u}(\varepsilon)$ to $\mathbf{u}^{0}$ in $\mathcal{V}$, we have only to prove that $u_{\alpha}(\varepsilon) \rightarrow u_{\alpha}^{0}$ in $H^{1}\left(\Omega^{m}\right)$. For this let us apply the classical Korn inequality to $\widehat{\mathbf{u}}(\varepsilon)=\left(\widehat{u}_{i}(\varepsilon)\right):=\left(u_{1}(\varepsilon), u_{2}(\varepsilon), 0\right)$ and $\widehat{\mathbf{u}}^{0}=$ $\left(\widehat{u}_{i}^{0}\right):=\left(u_{1}^{0}, u_{2}^{0}, 0\right)$. We set $\widehat{e}_{i j}(\mathbf{z}):=\frac{1}{2}\left(\partial_{i} z_{j}+\partial_{j} z_{i}\right)$ and we remark that from (vi) it follows easily : $\widehat{e}_{\alpha \beta}(\widehat{\mathbf{u}}(\varepsilon)) \rightarrow e_{\alpha \beta}\left(\mathbf{u}^{0}\right)$ in $L^{2}\left(\Omega^{m}\right)$. Hence, one has only to prove that $\widehat{e}_{\alpha 3}(\widehat{\mathbf{u}}(\varepsilon)) \rightarrow \widehat{e}_{\alpha 3}\left(\widehat{\mathbf{u}}^{0}\right)=\frac{1}{2} \partial_{3} u_{\alpha}^{0}$ in $L^{2}\left(\Omega^{m}\right)$. From $(i)$ and $(i i)$ we have that $u_{\alpha}(\varepsilon) \rightarrow u_{\alpha}^{0}$ in $L^{2}\left(\Omega^{m}\right)$ and $\partial_{3} u_{\alpha}^{0}=0$. Thus $\widehat{e}_{\alpha 3}(\widehat{\mathbf{u}}(\varepsilon))=\frac{1}{2} \partial_{3} u_{\alpha}(\varepsilon) \rightarrow 0$ in $H^{-1}\left(\Omega^{m}\right)$. Therefore we need only to prove that also $\partial_{\beta 3} u_{\alpha}(\varepsilon) \rightarrow 0$ in $H^{-1}\left(\Omega^{m}\right)$. One has

$$
\begin{aligned}
\partial_{\beta 3} u_{\alpha}(\varepsilon)= & \partial_{3}\left(\widehat{e}_{\alpha \beta}(\widehat{\mathbf{u}}(\varepsilon))+\partial_{\beta}\left(\varepsilon e_{\alpha 3}(\varepsilon ; \mathbf{u}(\varepsilon))+\varepsilon \Gamma_{\alpha 3}^{\sigma}(\varepsilon) u_{\sigma}(\varepsilon)\right)+\right. \\
& -\partial_{\alpha}\left(\varepsilon e_{\beta 3}(\varepsilon ; \mathbf{u}(\varepsilon))+\varepsilon \Gamma_{\beta 3}^{\sigma}(\varepsilon) u_{\sigma}(\varepsilon)\right) \\
& \longrightarrow \partial_{3}\left(e_{\alpha \beta}\left(\mathbf{u}^{0}\right)\right) \text { in } H^{-1}\left(\Omega^{m}\right)
\end{aligned}
$$

From $(i v)$ it follows that $\partial_{3}\left(e_{\alpha \beta}\left(\mathbf{u}^{0}\right)\right)=0$ and hence the announced strong convergence holds.

\subsection{Strong convergence for $p=3$}

Let $\mathbf{u}(\varepsilon) \in V$ be the solution of (6) for $p=3$. Thanks to the assumptions on the loading, the coercivity of the bilinear forms and Proposition 1 we can write the following a priori estimates:

$$
\begin{aligned}
& \|\mathbf{u}(\varepsilon)\|_{1, \Omega} \leq C \\
& \frac{1}{\varepsilon^{2}} \| e_{i j}\left(\varepsilon ; \mathbf{u}(\varepsilon) \|_{0, \Omega^{m}}^{2} \leq C .\right.
\end{aligned}
$$

Theorem 4 The sequence $(\mathbf{u}(\varepsilon))_{\varepsilon>0}$ converges strongly in $H^{1}\left(\Omega ; \mathbb{R}^{3}\right)$ to $\mathbf{u}^{0} \in$ $V_{F}$, the unique solution of problem (14). 
Proof For the sake of clarity, the proof is divided into five steps numbered from $(i)$ to $(v)$.

(i) From the a priori bound $\left(25_{1}\right)$ it follows that there exist $\mathbf{u} \in V$ and a subsequence not relabeled such that $\mathbf{u}(\varepsilon) \rightarrow \mathbf{u}$ in $H^{1}\left(\Omega ; \mathbb{R}^{3}\right)$. Estimate $\left(25_{2}\right)$ implies that $\frac{1}{\varepsilon} \partial_{3} u_{3}(\varepsilon) \rightarrow 0$ in $L^{2}\left(\Omega^{m}\right)$ and that $e_{\alpha 3}(\varepsilon ; \mathbf{u}(\varepsilon)) \rightarrow 0$ in $L^{2}\left(\Omega^{m}\right)$. Therefore

$$
\frac{1}{\varepsilon} \partial_{3} u_{\alpha}(\varepsilon) \rightarrow-\partial_{\alpha} u_{3}-2 b_{\alpha}^{\sigma} u_{\sigma} \text { in } L^{2}\left(\Omega^{m}\right),
$$

and $\partial_{3} \mathbf{u}=0$ in $\Omega^{m}$. From estimate $\left(25_{2}\right)$ we get that $e_{\alpha \beta}(\varepsilon ; \mathbf{u}(\varepsilon)) \rightarrow 0=$ $\gamma_{\alpha \beta}(\mathbf{u})$ in $L^{2}\left(\Omega^{m}\right)$. Thus $\mathbf{u} \in\left\{\mathbf{v} \in H^{1}\left(\Omega, \mathbb{R}^{3}\right): \partial_{3} \mathbf{v}=\mathbf{0}, \gamma_{\alpha \beta}(\mathbf{v})=0\right.$ in $\Omega^{m}, \mathbf{v}_{\mid \Gamma_{0}}=$ $\mathbf{0}\}$. At last, estimate $\left(25_{2}\right)$ yields the existence of $z_{i j} \in L^{2}\left(\Omega^{m}\right)$ such that $\frac{1}{\varepsilon} e_{i j}(\varepsilon ; \mathbf{u}(\varepsilon)) \rightarrow z_{i j}$ in $L^{2}\left(\Omega^{m}\right)$.

(ii) In order to prove that $u_{3} \in H^{2}\left(\Omega^{m}\right)$, let us define

$$
u_{\alpha}^{1}(\varepsilon)(\widetilde{x}):=\frac{1}{\varepsilon} \int_{-1}^{1} x_{3} u_{\alpha}(\varepsilon) d x_{3}=\frac{1}{2 \varepsilon} \int_{-1}^{1}\left(1-x_{3}^{2}\right) \partial_{3} u_{\alpha}(\varepsilon) d x_{3} .
$$

Then, using (i) it follows that

$$
\begin{aligned}
u_{\alpha}^{1}(\varepsilon) \rightarrow & \frac{1}{2} \int_{-1}^{1}\left(x_{3}^{2}-1\right)\left(\partial_{\alpha} u_{3}+2 b_{\alpha}^{\sigma} u_{\sigma}\right) d x_{3}= \\
& =-\frac{2}{3}\left(\partial_{\alpha} u_{3}+2 b_{\alpha}^{\sigma} u_{\sigma}\right) \text { in } L^{2}(\omega) .
\end{aligned}
$$

Actually, this convergence holds weakly in $H^{1}(\omega)$. Indeed, one has

$$
\begin{aligned}
& \frac{1}{2}\left(\partial_{\alpha} u_{\beta}^{1}(\varepsilon)+\partial_{\beta} u_{\alpha}^{1}(\varepsilon)\right)=\frac{1}{2 \varepsilon} \int_{-1}^{1} x_{3}\left(\partial_{\alpha} u_{\beta}(\varepsilon)+\partial_{\beta} u_{\alpha}(\varepsilon)\right) d x_{3}= \\
& =\int_{-1}^{1} x_{3} \frac{1}{\varepsilon} e_{\alpha \beta}(\varepsilon ; \mathbf{u}(\varepsilon)) d x_{3}+\frac{1}{2 \varepsilon} \int_{-1}^{1}\left(1-x_{3}^{2}\right)\left(\Gamma_{\alpha \beta}^{\sigma} \partial_{3} u_{\sigma}(\varepsilon)+b_{\alpha \beta} \partial_{3} u_{3}(\varepsilon)\right) d x_{3}+ \\
& -\int_{-1}^{1} x_{3}^{2} b_{\alpha}^{\sigma} b_{\sigma \beta} u_{3}(\varepsilon) d x_{3}+\frac{1}{\varepsilon} \int_{-1}^{1} x_{3}\left(\Gamma_{\alpha \beta}^{\sigma}(\varepsilon)-\Gamma_{\alpha \beta}^{\sigma}\right) u_{\sigma}(\varepsilon) d x_{3}
\end{aligned}
$$

The first term in the right-hand side converges weakly to $\int_{-1}^{1} x_{3} z_{\alpha \beta} d x_{3}$ in $L^{2}(\omega)$ and the second term tends to $-\frac{2}{3} \Gamma_{\alpha \beta}^{\sigma}\left(\partial_{\sigma} u_{3}+2 b_{\sigma}^{\tau} u_{\tau}\right)$ in $L^{2}(\omega)$. The last terms converge evidently in $L^{2}(\omega)$. By the classical Korn inequality it follows that the sequence $\left(u_{\alpha}^{1}(\varepsilon)\right)_{\varepsilon>0}$ is bounded in $H^{1}(\omega)$, hence $-\frac{2}{3}\left(\partial_{\alpha} u_{3}+2 b_{\alpha}^{\sigma} u_{\sigma}\right) \in$ $H^{1}(\omega)$ and so $\partial_{\alpha} u_{3} \in H^{1}(\omega)$. Thus $u_{3} \in H^{2}(\omega)$ and since $\partial_{3} u_{3}=0$, one has $u_{3} \in H^{2}\left(\Omega^{m}\right)$ so that $\mathbf{u} \in V_{F}$.

(iii) By multiplying (6) by $\varepsilon^{2}$ and by letting $\varepsilon \rightarrow 0$, we deduce that

$$
z_{33}=-\frac{A^{\alpha \beta 33}(0)}{A^{3333}(0)} z_{\alpha \beta} \text { and } z_{\alpha 3}=0
$$


Afterwards, by multiplying (6) by $\varepsilon$, by letting $\varepsilon \rightarrow 0$ and by choosing test functions such that $\partial_{3} v_{3}=0$, we obtain:

$$
\lim _{\varepsilon \rightarrow 0} \int_{\Omega^{m}} \frac{1}{\varepsilon} A^{\alpha 3 \sigma 3}(0) e_{\alpha 3}(\varepsilon ; \mathbf{u}(\varepsilon)) e_{\sigma 3}(\varepsilon ; \mathbf{v}) d x=-\int_{\Omega^{m}} a^{\alpha \beta \sigma \tau} z_{\alpha \beta} e_{\sigma \tau}^{0}(\mathbf{v}) d x
$$

Let us now remark that

$$
\begin{array}{r}
\partial_{3} e_{\alpha \beta}(\varepsilon ; \mathbf{u}(\varepsilon))=\varepsilon\left\{\partial_{\alpha} e_{\beta 3}(\varepsilon ; \mathbf{u}(\varepsilon))+\partial_{\beta} e_{\alpha 3}(\varepsilon ; \mathbf{u}(\varepsilon))-\partial_{\alpha \beta} u_{3}(\varepsilon)+\right. \\
\left.+\partial_{\beta}\left(\Gamma_{\alpha 3}^{\sigma}(\varepsilon) u_{\sigma}(\varepsilon)\right)+\partial_{\alpha}\left(\Gamma_{\beta 3}^{\sigma}(\varepsilon) u_{\sigma}(\varepsilon)\right)\right\}-\partial_{3}\left(\Gamma_{\alpha \beta}^{p}(\varepsilon) u_{p}(\varepsilon)\right)
\end{array}
$$

From (27) and recalling that $\left.b_{\beta}^{\tau}\right|_{\alpha}=\left.b_{\alpha}^{\tau}\right|_{\beta}$ it follows that in $H^{-1}\left(\Omega^{m}\right)$ one has

$$
\partial_{3} z_{\alpha \beta}=\lim _{\varepsilon \rightarrow 0} \frac{1}{\varepsilon} \partial_{3} e_{\alpha \beta}(\varepsilon ; \mathbf{u}(\varepsilon))=-\rho_{\alpha \beta}(\mathbf{u}) .
$$

However, since $u_{3} \in H^{2}\left(\Omega^{m}\right)$ the equality $\rho_{\alpha \beta}(\mathbf{u})=-\partial_{3} z_{\alpha \beta}$ holds in $L^{2}\left(\Omega^{m}\right)$. Given any $\boldsymbol{\eta} \in H^{1}\left(\omega, \mathbb{R}^{2}\right) \times H^{2}(\omega)$ such that $\gamma_{\alpha \beta}(\boldsymbol{\eta})=0$ let $\mathbf{v} \in H^{1}\left(\Omega^{m}, \mathbb{R}^{3}\right)$ defined by $v_{\alpha}=\eta_{\alpha}-x_{3} \theta_{\alpha}$ and $v_{3}=\eta_{3}$, where $\theta_{\alpha}:=\partial_{\alpha} \eta_{3}+2 b_{\alpha}^{\sigma} \eta_{\sigma}$ and $\boldsymbol{\theta}=$ $\left(\theta_{i}\right):=\left(\theta_{1}, \theta_{2}, 0\right)$. Since then $e_{\sigma 3}(\varepsilon ; \mathbf{v})=\frac{1}{2}\left(-\frac{1}{\varepsilon} \theta_{\alpha}+\partial_{\alpha} \eta_{3}\right)-\Gamma_{\alpha 3}^{\sigma}(\varepsilon)\left(\eta_{\sigma}-x_{3} \theta_{\sigma}\right)$, $z_{\alpha 3}=0$ and $e_{\sigma \tau}^{0}(\mathbf{v})=-x_{3} e_{\sigma \tau}^{0}(\boldsymbol{\theta})$, we obtain from (26)

$$
\begin{aligned}
\lim _{\varepsilon \rightarrow 0} \frac{1}{\varepsilon^{2}} \int_{\Omega^{m}} A^{\alpha 3 \sigma 3}(0) e_{\alpha 3}(\varepsilon, \mathbf{u}(\varepsilon)) & \frac{1}{2} \theta_{\sigma} d x= \\
=\int_{\Omega^{m}} a^{\alpha \beta \sigma \tau} z_{\alpha \beta} e_{\sigma \tau}^{0}(\mathbf{v}) d x= & -\int_{\Omega^{m}} a^{\alpha \beta \sigma \tau} z_{\alpha \beta} x_{3} e_{\sigma \tau}^{0}(\boldsymbol{\theta}) d x \\
& =-\frac{1}{2} \int_{\Omega^{m}} a^{\alpha \beta \sigma \tau}\left(1-x_{3}^{2}\right) \partial_{3} z_{\alpha \beta} e_{\sigma \tau}^{0}(\boldsymbol{\theta}) d x \\
& =\frac{1}{2} \int_{\Omega^{m}}\left(1-x_{3}^{2}\right) a^{\alpha \beta \sigma \tau} \rho_{\alpha \beta}(\mathbf{u}) e_{\sigma \tau}^{0}(\boldsymbol{\theta}) d x
\end{aligned}
$$

(iv) When $\mathbf{v} \in V_{F}$ one has

$$
\left\{\begin{array}{l}
e_{\alpha \beta}(\varepsilon ; \mathbf{v})=\varepsilon x_{3}\left(\left.b_{\beta}^{\sigma}\right|_{\alpha} v_{\sigma}+b_{\alpha}^{\sigma} b_{\sigma \beta} v_{3}\right)+O\left(\varepsilon^{2}\right) \\
e_{\alpha 3}(\varepsilon ; \mathbf{v})=\frac{1}{2} \partial_{\alpha} v_{3}+b_{\alpha}^{\sigma} v_{\sigma}+O(\varepsilon) \\
e_{33}(\varepsilon ; \mathbf{v})=0
\end{array}\right.
$$

Hence if we choose in $(6) \mathbf{v} \in V_{F}$, by passing to the limit we get:

$$
\begin{aligned}
A^{+}(\mathbf{u}, \mathbf{v})+ & A^{-}(\mathbf{u}, \mathbf{v})+\int_{\Omega^{m}} a^{\alpha \beta \sigma \tau} z_{\alpha \beta} x_{3}\left(\left.b_{\tau}^{\mu}\right|_{\sigma} v_{\mu}+b_{\sigma}^{\mu} b_{\mu \tau} v_{3}\right) d x+ \\
& +\lim _{\varepsilon \rightarrow 0} \frac{1}{\varepsilon^{2}} \int_{\Omega^{m}} A^{\alpha 3 \sigma 3}(0) e_{\sigma 3}(\varepsilon ; \mathbf{u}(\varepsilon))\left(\frac{1}{2} \partial_{\alpha} v_{3}+b_{\alpha}^{\tau} v_{\tau}\right) d x=L(\mathbf{v}) .
\end{aligned}
$$

Let $\xi_{\alpha}:=\partial_{\alpha} v_{3}+2 b_{\alpha}^{\tau} v_{\tau}$ and $\boldsymbol{\xi}=\left(\xi_{i}\right):=\left(\xi_{1}, \xi_{2}, 0\right)$. Then, by using (28) and the relation $e_{\sigma \tau}^{0}(\boldsymbol{\xi})-\left.b_{\tau}^{\mu}\right|_{\sigma} v_{\mu}-b_{\sigma}^{\mu} b_{\mu \tau} v_{3}=\rho_{\sigma \tau}(\mathbf{v})$, we obtain that

$$
A^{+}(\mathbf{u}, \mathbf{v})+A^{-}(\mathbf{u}, \mathbf{v})+\frac{2}{3} \int_{\omega} a^{\alpha \beta \sigma \tau} \rho_{\alpha \beta}(\mathbf{u}) \rho_{\sigma \tau}(\mathbf{v}) d \widetilde{x}=L(\mathbf{v})
$$


so that $\mathbf{u}=\mathbf{u}^{0}$, the one and only one solution of problem (14).

$(v)$ It remains to prove the strong convergence. Let $\left(\phi^{\eta}\right)_{\eta>0} \subset V$ be defined as follows:

$$
\begin{cases}\phi_{\alpha}^{\eta}(x)=x_{3}^{2}\left(b_{\alpha}^{\sigma} \partial_{\sigma} u_{3}^{0}(\widetilde{x})+b_{\alpha}^{\sigma} b_{\sigma}^{\tau} u_{\tau}^{0}(\widetilde{x})\right) & \text { for all } x \in \Omega^{m} \\ \phi_{3}^{\eta}(x)=\frac{x_{3}^{2}}{2} w^{\eta}(\widetilde{x}) & \text { for all } x \in \Omega^{m}\end{cases}
$$

where $\left(w^{\eta}\right)_{\eta>0}$ is a sequence in $\mathcal{D}(\omega)$ which satisfies in $L^{2}(\omega)$

$$
w^{\eta} \rightarrow \frac{A^{\alpha \beta 33}(0)}{A^{3333}(0)} \rho_{\alpha \beta}\left(\mathbf{u}^{0}\right) \text {. }
$$

Let $\psi \in V$ be such that

$$
\left\{\begin{array}{ll}
\psi_{\alpha}(x)=-x_{3}\left(\partial_{\alpha} u_{3}^{0}(\widetilde{x})+2 b_{\alpha}^{\tau} u_{\tau}^{0}(\widetilde{x})\right) & \text { for all } x \in \Omega^{m} \\
\psi_{3}(x)=0 & \text { for all } x \in \Omega^{m}
\end{array} .\right.
$$

Then $\mathbf{u}(\varepsilon)-\mathbf{u}^{0}-\varepsilon \boldsymbol{\psi}-\varepsilon^{2} \boldsymbol{\phi}^{\eta} \in V$ and

$$
\begin{aligned}
& e_{\alpha \beta}\left(\varepsilon ; \mathbf{u}^{0}-\varepsilon \boldsymbol{\psi}-\varepsilon^{2} \boldsymbol{\phi}^{\eta}\right)=-\varepsilon x_{3} \rho_{\alpha \beta}\left(\mathbf{u}^{0}\right)+O\left(\varepsilon^{2}\right) \\
& e_{\alpha \beta}\left(\varepsilon ; \mathbf{u}^{0}-\varepsilon \boldsymbol{\psi}-\varepsilon^{2} \boldsymbol{\phi}^{\eta}\right)=O\left(\varepsilon^{2}\right) \\
& e_{\alpha \beta}\left(\varepsilon ; \mathbf{u}^{0}-\varepsilon \boldsymbol{\psi}-\varepsilon^{2} \boldsymbol{\phi}^{\eta}\right)=\varepsilon x_{3} w^{\eta}
\end{aligned}
$$

where the order symbol $O\left(\varepsilon^{2}\right)$ is meant with respect to the norm of $L^{2}\left(\Omega^{m}\right)$. Setting $A(\cdot, \cdot):=A^{+}(\cdot, \cdot)+A^{-}(\cdot, \cdot)+\varepsilon A^{m}(\cdot, \cdot)$, by virtue of the coercivity we obtain :

$A\left(\mathbf{u}(\varepsilon)-\mathbf{u}^{0}-\varepsilon \boldsymbol{\psi}-\varepsilon^{2} \boldsymbol{\phi}^{\eta}, \mathbf{u}(\varepsilon)-\mathbf{u}^{0}-\varepsilon \boldsymbol{\psi}-\varepsilon^{2} \boldsymbol{\phi}^{\eta}\right) \geq C\left\|\mathbf{u}(\varepsilon)-\mathbf{u}^{0}-\varepsilon \boldsymbol{\psi}-\varepsilon^{2} \boldsymbol{\phi}^{\eta}\right\|_{V}^{2}$.

By letting $\varepsilon \rightarrow 0$ and by using a standard diagonalization argument, one has

$$
\begin{aligned}
& \lim _{\eta \rightarrow 0} \lim _{\varepsilon \rightarrow 0} A\left(\mathbf{u}(\varepsilon)-\mathbf{u}^{0}-\varepsilon \boldsymbol{\psi}-\varepsilon^{2} \boldsymbol{\phi}^{\eta}, \mathbf{u}(\varepsilon)-\mathbf{u}^{0}-\varepsilon \boldsymbol{\psi}-\varepsilon^{2} \boldsymbol{\phi}^{\eta}\right)= \\
& =L\left(\mathbf{u}^{0}\right)-A^{+}\left(\mathbf{u}^{0}, \mathbf{u}^{0}\right)-A^{-}\left(\mathbf{u}^{0}, \mathbf{u}^{0}\right)-\int_{\Omega^{m}} x_{3}^{2} a^{\alpha \beta \sigma \tau} \rho_{\alpha \beta}\left(\mathbf{u}^{0}\right) \rho_{\sigma \tau}\left(\mathbf{u}^{0}\right) d x=0
\end{aligned}
$$

which completes the proof.

Acknowledgement. The work described in this paper has been developed during the A.-L. Bessoud permanence at Laboratoire de Mécanique et Génie Civil , Université Montpellier II, as part of her Ph.D thesis entitled "Modélisation mathématique d'un multi-matériau" and during the M. Serpilli permanence at I.N.R.I.A. - Rocquencourt, supported by the French Agence Nationale de la Recherche (ANR) under grant epsilon (BLAN08-2-312370): Domain decomposition and multi-scale computations of singularities in mechanical structures. 


\section{References}

1. Bathe, K.J., Chapelle, D., The finite element analysis of shells-fundamentals, Springer, Berlin (2003)

2. Bessoud, A.-L., Krasucki, F., Michaille, G., "Multi-materials with strong interface: variational modelings", Asymptot. Anal., 61, 1-19 (2008).

3. Brezis, H., Caffarelli, L.A., Friedman, A., "Reinforcement problems for elliptic equations and variational inequalities", Ann. Mat. Pura Appl., 4, 123, 219-246 (1980).

4. Caillerie, D.,"The effect of a thin inclusion of high rigidity in an elastic body", Math. Methods Appl. Sci., 2, 251-270 (1980).

5. Ciarlet, P.G., Mathematical Elasticity, vol. III: Theory of shells, Studies in mathematics and its applications, North-Holland, Amsterdam (2000).

6. Ciarlet P.G., Le Dret H., Nzengwa R. ," Junctions between three-dimensional and twodimensional linearly elastic structures", J. Math. Pures Appl., 68, 261-295,(1989).

7. Chapelle, D., Ferent, A., "Modeling of the inclusion of a reinforcing sheet within a $3 \mathrm{D}$ medium", Math. Models Methods Appl. Sci., 13, 573-595 (2003).

8. Geymonat, G., Krasucki, F., Lenci., S. "Mathematical analysis of a bounded joint with a soft thin adhesive", Math. Mech. Solids, 4, 201-225, (1999).

9. Le Dret H., Problemes Variationnels dans les Multi-Domaines, Masson, Paris, (1991).

10. Naghdi P.M. , "Foundations of elastic shell theory", Progress in Solid Mechanics, 4, 1-90 North-Holland, Amsterdam (1963).

11. Pham Huy, H., Sanchez-Palencia, E., "Phénomène de transmission à travers des couches minces de conductivité élevée", J. Math. Anal. Appl., 47, 284-309 (1974).

12. Sanchez-Hubert, J., Sanchez-Palencia, E., Coques Elastiques Minces. Propriétés Asymptotiques, Masson (1997). 